\title{
Lunar magnetic field models from Lunar Prospector and SELENE/Kaguya along-track magnetic field gradients
}

Ravat, D.; Purucker, M.E.; Olsen, N.

Published in:

Journal of Geophysical Research: Planets

Link to article, DOI:

10.1029/2019JE006187

Publication date:

2020

Document Version

Peer reviewed version

Link back to DTU Orbit

Citation (APA):

Ravat, D., Purucker, M. E., \& Olsen, N. (2020). Lunar magnetic field models from Lunar Prospector and SELENE/Kaguya along-track magnetic field gradients. Journal of Geophysical Research: Planets, 125(7), [e2019JE006187]. https://doi.org/10.1029/2019JE006187

\section{General rights}

Copyright and moral rights for the publications made accessible in the public portal are retained by the authors and/or other copyright owners and it is a condition of accessing publications that users recognise and abide by the legal requirements associated with these rights.

- Users may download and print one copy of any publication from the public portal for the purpose of private study or research.

- You may not further distribute the material or use it for any profit-making activity or commercial gain

- You may freely distribute the URL identifying the publication in the public portal 
Ravat Dhananjay (Orcid ID: 0000-0003-1962-4422)

Olsen Nils (Orcid ID: 0000-0003-1132-6113)

\title{
Lunar magnetic field models from Lunar Prospector and SELENE/Kaguya along-track magnetic field gradients
}

\author{
D. Ravat ${ }^{1}$, M. E. Purucker ${ }^{2}$, and N. Olsen ${ }^{3}$ \\ University of Kentucky, Lexington, Kentucky, USA \\ ${ }^{2}$ NASA-GSFC, Greenbelt, Maryland, USA \\ ${ }^{3}$ Technical University of Denmark, Kongens Lyngby, Denmark.
}

Corresponding author: D. Ravat (dhananjay.ravat@uky.edu)

\section{Key Points:}

- New high resolution surface vector magnetic field models are derived from crustal sources from Lunar Prospector satellite observations

- Along-orbit gradients of vector field measurements alone (excluding vector fields) lead to significant reduction in the external fields

- The effectiveness of equivalent monopoles vs dipoles and least-squares vs sparse matrix inversion techniques is evaluated

\section{Plain Language Summary:}

The Moon has magnetic field variations (anomalies) caused by permanently magnetized rocks formed during the era of its early strong core field dynamo. High resolution maps of magnetic anomalies allow us to investigate the depths, shapes, and nature of the sources and conjecture the origin of these individual anomaly features. Magnetization direction of these permanently magnetized sources also tells us if the Moon's rotational axis has changed its position during the time period when the core magnetic field dynamo was active. The inferred magnetization direction of a large magnetic anomaly in the Serenitatis impact basin (nearside) suggests that the Moon may have changed its orientation significantly (more than

This article has been accepted for publication and undergone full peer review but has not been through the copyediting, typesetting, pagination and proofreading process which may lead to differences between this version and the Version of Record. Please cite this article as doi: 10.1029/2019JE006187 
$\left.45^{\circ}\right)$ since the formation of the basin. Using magnetometer data from Lunar Prospector (NASA) and Kaguya (Japan) satellites, we use methods of reconstructing the field at the lunar surface, which in turn will allow investigations on the origin of other similar features.

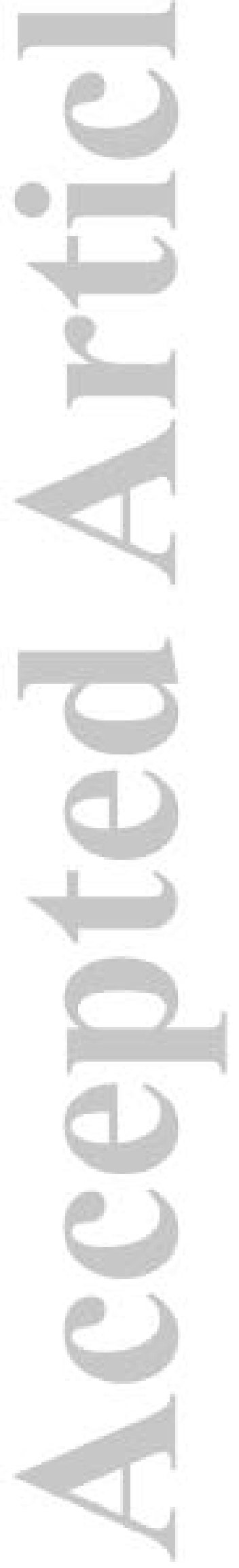




\begin{abstract}
We use L1-norm model regularization of $\left|\mathrm{B}_{\mathrm{r}}\right|$ component at the surface on magnetic monopoles bases and along-track magnetic field differences alone (without vector observations) to derive high quality global magnetic field models at the surface of the Moon. The practical advantages to this strategy are: monopoles are more stable at closer spacing in comparison to dipoles, improving spatial resolution; L1-norm model regularization leads to sparse models which may be appropriate for the Moon which has regions of localized magnetic field features; and along-track differences reduce the need for ad-hoc external field noise reduction strategies. We examine also the use of Lunar Prospector (LP) and SELENE/Kaguya magnetometer data, combined and separately, and find that the LP alongtrack vector field differences lead to surface field models that require weaker regularization and, hence, result in higher spatial resolution. Significantly higher spatial resolution (wavelengths of roughly $25-30 \mathrm{~km}$ ) and higher amplitude surface magnetic fields can be derived over localized regions of high amplitude anomalies (due to their higher signal-tonoise ratio). These high resolution field models are also compared with the results of Surface Vector Mapping (SVM) approach of Tsunakawa et al. (2015). Finally, the monopoles- as well as dipoles-based patterns of the Serenitatis high amplitude magnetic feature have characteristic textbook patterns of $\mathrm{B}_{\mathrm{r}}$ and $\mathrm{B}_{\theta}$ component fields from a nearly vertically downwardly magnetized source region and it implies that the principal source of the anomaly was formed when the region was much closer to the north magnetic pole of the Moon.
\end{abstract}

\title{
1 Introduction
}

The discovery of a $38 \mathrm{nT}$ magnetic field at the Apollo 12 site and later static fields from Apollo 14, 15, and 16 sites (up to $327 \mathrm{nT}$ at Apollo 16) and fields measured by Apollo sub-satellites forced researchers to reject the concept of a non-magnetic Moon (Daily \& Dyal, 1979; Dyal et al., 1974; Sharp et al., 1973). The consideration of the role of remanent crustal magnetism in shaping lunar magnetic fields was confirmed by significant natural remanent magnetization of samples returned from Apollo and Luna 16 missions (Collinson et al., 1973; Nagata et al., 1971; Runcorn et al., 1970; Strangway et al., 1970). A recent comprehensive study of the samples, however, suggests that their magnetization may be about factor of 3 smaller than originally measured (Lepaulard et al., 2019), but it is still quite significant (up to about $0.75 \mathrm{~A} / \mathrm{m}$ ) and susceptibilities as high as $0.045 \mathrm{SI}$ units (using basalt density of 3200 $\mathrm{kg} / \mathrm{m}^{3}$, Kiefer et al., 2012).

Lunar Prospector (LP) (1998-1999) was the first spacecraft to globally survey the Moon's magnetic field (Hood et al., 2001; Lin et al., 1998) and more recently Japanese SELENE/Kaguya mission collected magnetic data from 2007 to 2009 (Takahashi et al., 2009). These two orbital datasets, in conjunction with the study of samples, form the basis for contemporary global analysis of lunar magnetism. Analysis of these datasets using advanced data reduction and modeling techniques (Purucker \& Nicholas, 2010; Tsunakawa et al., 2015) have led to numerous regional studies and interpretations (e.g., Arkani-Hamed \& Boutin, 2014, 2017; Hemingway \& Garrick-Bethell, 2012; Nayak et al., 2017; Oliveira \& Wieczorek, 2017; Purucker et al., 2012; Wieczorek et al., 2012; Wieczorek, 2018).

Despite these studies, most sources of lunar magnetic anomalies remain enigmatic: e.g., their association with lunar swirls, which are bright surface regions where solar wind particles are deflected by lunar magnetic field and where the intra-swirl "dark lanes" 
correspond to locations where the field-lines are open and where the solar wind can directly hit the surface (Hood \& Schubert, 1980; Denevi et al., 2016); magnetic sources in South Pole - Aitken (SPA) basin region, which are interpreted to be meteoritic ejecta material by Wieczorek et al. (2012), and post-impact magmatic intrusions/lava ponds by Purucker et al. (2012); melt sheets in Nectarian impact basins (Hood, 2011, Oliveira et al., 2017). Some of the interpretational aspects are hindered by the inability of satellite-altitude data in capturing short-wavelength field variations $(<20-30 \mathrm{~km}$ wavelength, roughly corresponding to the altitude at which the data were taken) and some due to errors in the field models themselves. In addition to these difficulties, a significant amount of anomaly superposition and coalescence must occur and information critical to the interpretation of near-surface and small dimension magnetic sources is lost.

An advantage of using the gradients is that they make perceptible some of the shorter wavelength information useful in interpretation and, under ideal conditions (i.e., orbits nearpendicular to two-dimensional sources), they can also be directly used in interpretation methods that use derivatives of fields (e.g., see several methods of interpretation discussed in Blakely, 1995). In modeling the fields themselves, gradients help in removing the deleterious effect of long-wavelength orbital residuals introduced by large-scale external field contributions as demonstrated by Olsen et al. (2017). For convenience, we use the terms 'along-track differences' of observations (which are scaled approximations of gradients) and 'gradients' synonymously in the manuscript. So far direct observations of gradients have not been made on the Moon.

In this study, we present new vector gradient based models of crustal magnetic field at the lunar surface with data from the Lunar Prospector (LP) satellite using global and local sets of magnetic equivalent sources (monopoles, cf. O'Brien \& Parker, 1994; Olsen et al., 2017). We use the scheme of iteratively reweighted least squares to account for non-Gaussian data errors. This is followed by L1-norm model regularization with constraints in which the amplitudes of these monopoles are determined by minimizing the misfit to the along-track differences of components together with the average of $\left|\mathrm{B}_{\mathrm{r}}\right|$ at the Moon's ellipsoid surface (i.e. applying a L1-norm model regularization of $\left|\mathrm{B}_{\mathrm{r}}\right|$ ). In deriving our preferred field models, we did not use vector fields themselves because external field contamination led to spurious anomalies in the downward continued field models even with stringent data selection criteria and ad-hoc noise removal techniques.

During the study, we also examined permutations of different data selection criteria along with using low-altitude vector component and along-track gradient data from LP and SELENE, separately and in various combinations. We found that, with the current datasets, models based on low-altitude LP along-track gradients alone with minimal processing were superior to other variants. The currently available SELENE/Kaguya extended mission (lowaltitude) data from the Japan Aerospace Exploration Agency's (JAXA) data portal suffer from positioning inaccuracies of several meters to kilometers (Goossens et al., 2020); however, the positions have been improved recently by refining orbit solutions (Goossens et al., 2020, and can be found at https://pgda.gsfc.nasa.gov/products/74). Using these improved orbital positions, we re-determined our models, but they did not lead to any noticeable definitive improvement in the structure or resolution of the fields. There are also other failure issues and differences between the Lunar Prospector and SELENE/Kaguya mission data as enumerated in section 7. Therefore, our preferred models rely solely on LP data. 
Several areas of the Moon have relatively stronger magnetic features than others and thus it was difficult to create global high spatial resolution surface vector field maps with the same regularization. Tsunakawa et al. (2015) used different amounts of regularization in different regions in order to create global maps (e.g., Tsunakawa et al., 2015); however, we chose to create higher resolution maps by optimizing regularization for key regions such as Reiner Gamma swirl, Serenitatis impact basin, and Von Kármán basin.

\section{The modeling methods}

\subsection{Monopoles for magnetic field mapping}

O'Brien and Parker (1994) first proposed the use of monopole basis functions for mapping global crustal/lithospheric magnetic fields. Even though the dipole formulations (Langlais et al., 2004; Mayhew, 1979; von Frese et al., 1981a; Dyment \& Arkani-Hamed, 1998) and spherical harmonic expansions (Langel \& Hinze, 1998; Maus et al., 2002; Maus, 2010) or their regional spherical cap variants (e.g. Haines, 1985; Thébault et al., 2006, Thébault, 2008) are customary for this purpose, the former suffers from instabilities due to close spacing of dipoles (Langlais et al., 2004; Mayhew, 1979; Ravat et al., 1991) and all methods suffer from limitations in computing power to variable extent. Monopoles can be placed relatively closer and shallower than dipoles to obtain stable solutions and thus can lead to improved spatial resolution. Recently, using the monopoles approach, Kother et al. (2015) and Olsen et al. (2017) have determined high resolution maps of the Earth's lithospheric magnetic field using CHAMP and Swarm satellite missions datasets. In the context of mapping the lunar magnetic field from SELENE/Kaguya and LP magnetic field observations, Tsunakawa et al. $(2010,2015)$ describe the surface vector mapping (SVM) method, which uses all three components of the magnetic field at the observation location to determine the radial component of the field at the surface.

In terms of the ability of along-track gradients to map the field, one only needs to determine the potential from the $\mathrm{B}_{\mathrm{r}}$ component. The knowledge of the radial derivative of potential on a sphere allows determination of Laplacian potential of internal origin (Backus et al., 1996). Similarly, the knowledge of the second radial derivative (or more generally, a radial derivative of any order) also determines the potential. We show in the supporting information (Figures $\mathrm{S} 1$ and $\mathrm{S} 2$ ) a model study demonstrating the recovery of $\mathrm{B}_{\mathrm{r}}$ component at the surface from the monopoles inversion of the $30 \mathrm{~km}$ altitude $\mathrm{N}-\mathrm{S}$ differences (i.e., simulated along-track gradients) by joint analysis of all three components together or by analyzing the individual components $\mathrm{B}_{\mathrm{r}}, \mathrm{B}_{\theta}$, and $\mathrm{B}_{\phi}$ separately. With the three components $\left(\Delta \mathrm{B}_{\mathrm{r}}, \Delta \mathrm{B}_{\theta}\right.$, and $\left.\Delta \mathrm{B}_{\phi}\right)$ or $\Delta \mathrm{B}_{\mathrm{r}}$ only inversions from $30 \mathrm{~km}$ altitude, one can recover nearly all of the information, except the shortest wavelengths of the field that are coalesced, attenuated, and related to round-off errors.

\subsection{Least-squares minimization of data residuals and L2- and L1-norm model regularization}

Using basis functions (dipoles, monopoles, spherical harmonic functions) that map the field using least-squares minimization of the residual between the observed and the modeled fields is the most common approach in magnetic field modeling. To mitigate noise in the downward continued fields, one can use additional information in the form of a constraint (e.g., squared length of the model vector or $\mathrm{B}_{\mathrm{r}}{ }^{2}$ averaged over the planetary surface), 
implemented as regularization (e.g., Kother et al., 2015; Maus et al., 2002; Thébault et al., 2006; Tsunakawa et al., 2015; Whaler, 1994). Purely L2 regularizations yield smoother solutions with source strengths distributed over larger areas (i.e., they are non-sparse). In sparse models, observations are explained with fewer model parameters and model parameters unessential for explaining data are removed during iterations.

Instead of minimization of the average of the squared length (the Euclidean norm), e.g., average of $\mathrm{B}_{\mathrm{r}}{ }^{2}$ at surface, one may also use other norms of minimizing the length (e.g., the average of $\left|\mathrm{B}_{\mathrm{r}}\right|$, etc.). This approach, which leads to sparse solutions, has been used by Morschhauser et al. (2014); Moore \& Bloxham (2017); and Olsen et al. (2017). The approach may also be desirable for the lunar magnetic field mapping because the Moon's field appears to be localized and has a number of regions without any significant observed fields. L1-norm model regularization is typically obtained iteratively using an approach known as Iteratively Reweighted Least Squares (IRLS), as described, for example, in Farquharson \& Oldenburg (1998). The iterative process requires a reasonable starting solution, here taken from the L2norm model regularized solution.

In this study, we used the approach of Olsen et al. (2017) which is described in detail in that manuscript and we refer readers interested in the details to it. Briefly, for the solution of L2-norm model regularization, we minimize the following cost function $(\Phi)$ using iteratively reweighted least-squares,

$$
\Phi=\mathbf{e}^{\mathrm{T}} \mathbf{W}_{\mathrm{d}} \mathbf{e}+\alpha^{2} \mathbf{m}^{\mathrm{T}} \mathbf{R} \mathbf{m},
$$

where $\mathbf{e}=\mathbf{d}-\mathbf{G m}$ is the data misfit vector (in which $\mathbf{d}$ is data vector, $\mathbf{m}$ is the model vector, $\mathbf{G}$ is the kernel relating model vector to data predictions), $\mathbf{W}_{\mathrm{d}}$ is the diagonal data weight matrix with elements $\mathrm{w} / \sigma^{2}$ (where $\sigma^{2}$ are the data variances, and $\mathrm{w}$ are the robust data weights), $\mathbf{R}$ is a model regularization matrix which results in the minimization of the global average of $\mathrm{B}_{\mathrm{r}}{ }^{2}$ at the surface of ellipsoid. The parameter $\alpha^{2}$ controls the relative contribution of the model regularization norm to the cost function. In iteratively reweighted least-squares, data weights " $w$ " were defined by Tukey's bi-weight function with the tuning constant $\mathrm{c}=$ 4.5 , which is close to the value of the statistically most efficient parameter for weighting residuals and removing outliers in robust regression (Constable, 1988; Farquharson \& Oldenberg, 1998).

The model regularization matrix $\mathbf{R}$ is determined using the relationship of the model parameters to $\mathrm{B}_{\mathrm{r}}$ over a distribution of points on the globe comparable to the number of model parameters. The relationship matrix is given as $\mathbf{b}=\left\{\mathrm{B}_{\mathrm{r}}\right\}=\mathbf{A}_{\mathrm{r}} \mathbf{m}$. For the L2-norm model regularized solution we use $\mathbf{R}=\mathbf{A}_{\mathrm{r}}^{\mathrm{T}} \mathbf{A}_{\mathrm{r}}$, taking into account the minimization of the global average of $\mathrm{B}_{\mathrm{r}}{ }^{2}$ at the surface of ellipsoid. On the other hand, the L1-norm model regularization constraint is implemented iteratively using a regularization matrix $\mathbf{R}=\mathbf{A}_{\mathrm{r}}^{\mathrm{T}} \mathbf{W}_{\mathrm{m}}$ $\mathbf{A}_{\mathrm{r}}$, where $\mathbf{W}_{\mathrm{m}}$ is the diagonal matrix of model parameter weights based on $\left|B_{\mathrm{r}}\right|$, and $\mathbf{R}$ is updated at each iteration to implement the L1-norm model regularization.

We used two variations of the approach for the global inversions: 35000 monopoles with $30 \mathrm{~km}$ equal-area spacing (equal-area spacing of sources using the algorithm of Leopardi, 2006) for global models, and 100000 monopoles (20 km spacing) in 84 subsets with $10^{\circ}$ overlap with neighboring regions such that the subsets could be merged in the center of the overlap region without edge effects. We used also different monopole depths to 
examine the stability of solutions at different monopole spacings and finally chose $20 \mathrm{~km}$ depth for the monopoles with horizontal spacing on the order of $20-30 \mathrm{~km}$. At smaller horizontal spacing of sources, smaller depths were acceptable but that is only feasible for smaller regions of investigation. The regularization parameter (or damping parameter), $\alpha$, was chosen based on visually stable appearance of the fields at the surface of the ellipsoid representing the Moon (see Figure S5 showing along-track trends in the supporting information which are inadequately regularized). In general, the optimum regularization parameter depends on the level of noise in the data as well as the equivalent source spacing. Using formulas in Olsen et al. (2017), monopole amplitudes can be converted into spherical harmonic coefficients and these were used in deriving formal variances of spherical harmonic coefficients (Olsen et al., 2017) used in evaluating the relative performance of LP and SELENE/Kaguya data based global field models up to degree/order 150 (see section 4.6).

\section{Data}

\subsection{Lunar Prospector magnetic field data}

We used five second data (roughly $0.27^{\circ}$ along orbit) from Lunar Prospector spacecraft available at NASA's Planetary Data System (PDS) from its extended mission (1 January to 28 July 1999, at altitudes between 12 and $48 \mathrm{~km}$ ). The PDS data were converted to latitude, longitude, altitude and $\mathrm{B}_{\mathrm{r}}, \mathrm{B}_{\theta}, \mathrm{B}_{\phi}$ components ( $\mathrm{r}$ - outward, $\theta$-southward, and $\phi$ -eastward as in the usual spherical coordinate system). We processed these data in multiple ways, and eventually settled on datasets either from the lunar wake with respect to the solar wind or in the Earth's magnetotail when the spacecraft was within $20^{\circ}$ with respect to the opposite side of the Sun (similar to Purucker \& Nicholas, 2010). We also used their procedure to fit and remove lunar internal and external field dipole terms (Purucker \& Nicholas, 2010). The wake/tail selection is important because crustal magnetic field lines are significantly compressed due to solar wind pressure (similar to pressure balance at the bow shock, de Pater \& Lissauer, 2015; Hood \& Schubert, 1980) for data taken directly in the solar wind. In models with vector component data, we also used ad-hoc procedures to obtain the cleanest possible data subset (e.g., up to $3^{\text {rd }}$ order polynomial removal, equivalent dipole based altitude-normalized cross-validation of fields from nearby pass segments, and then further removal of inconsistent pass segments identified manually). The models with vector data have N-S artifacts as shown in the supporting information Figure S5 unless they are heavily damped, which makes their anomalies subdued, and thus they are not our preferred models. In models where we used only along-track vector component differences, we did not use any ad-hoc procedures because they were not necessary as evident from along-track differences of $\mathrm{B}_{\mathrm{r}}$ component shown in Figure 1. In our wake/tail selected low-altitude data subset, there are $>1$ million points each of vector and along-track vector gradient observations (at altitudes $\leq 48 \mathrm{~km}$ ). In the polar regions however, we used all of the polar orbital segments beyond $\pm 75^{\circ}$ of latitude poleward as the wake selection ended up removing significant amount of polar data. Br component data from Tsunakawa et al. (2015) selection, comparable to Figure 1a, is shown in Figure S3 (supporting information). 

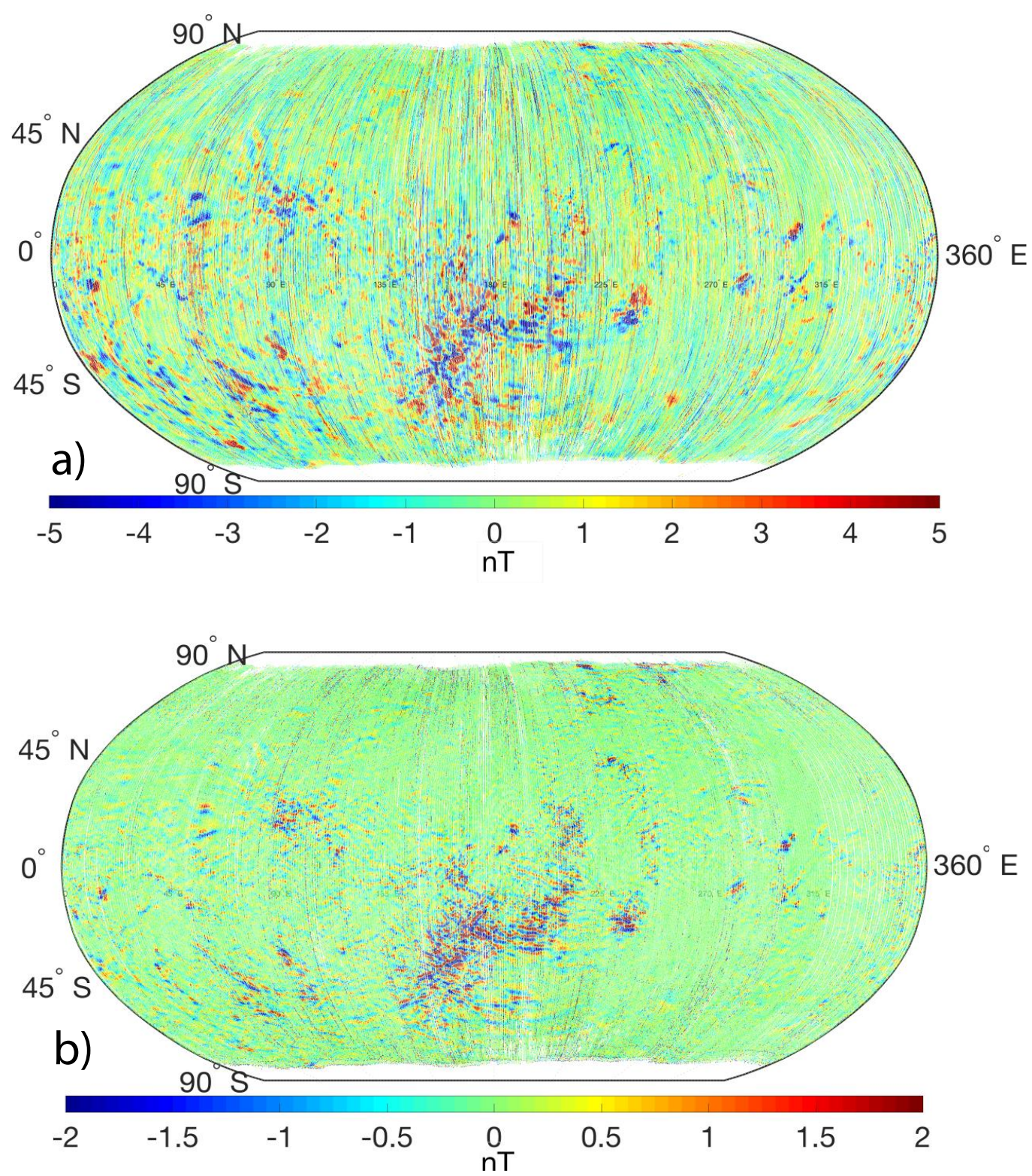

Figure 1. Scatterplots of Lunar Prospector satellite low-altitude $(\leq 48 \mathrm{~km})$ a) Br component from $\mathrm{S}-\mathrm{N}$ going (ascending) passes. The data in this figure are selected from the lunar wake region with respect to the solar wind and de-trended using a $3^{\text {rd }}$ order polynomial; b) five second along-track differences, $\Delta \mathrm{B}_{\mathrm{r}}$. There are still a few remaining orbital segment biases in part $b$ (which are differing levels of vector fields in neighboring orbits caused by external fields or instrument offsets), and these are treated in the inversion using variances of along-track differences and regularization. The data in part $b$ are selected from the lunar wake region with respect to the solar wind and without applying any de-trending or ad-hoc data selection. Robinson projection.

\subsection{SELENE/Kaguya magnetic field data}

We used the same processing scheme for SELENE/Kaguya low-altitude data from its extended mission. The SELENE/Kaguya crustal field data at the JAXA portal are at 4 second interval $\left(0.2^{\circ}\right.$ along-orbit spacing). These data are broadly similar to the LP data as shown 
from spatial comparisons by Tsunakawa et al. (2014). There are $>1.1$ million data points in selected vector fields and along-track field differences which range in altitude from 8 to 63 $\mathrm{km}$ (from 18 January to 8 June 2009). However, the bulk of these data are at altitudes $>35$ $\mathrm{km}$. Truly low altitude SELENE/Kaguya data are only present in and around South Pole Aitken basin and up to northern mid-latitudes in a longitude swath from $90^{\circ} \mathrm{E}$ to $265^{\circ} \mathrm{E}$. Along-track differences of $\mathrm{B}_{\mathrm{r}}$ component from the location corrected SELENE/Kaguya extended mission data (Goossens et al., 2020) processed identically to the LP data are shown in Figure 2 (for comparison with LP along-track differences in Figure 1). Despite these improved SELENE/Kaguya orbits, the results and analysis in section 4 show that the LP data subset performed better than the SELENE/Kaguya dataset in low spherical harmonic degrees and orders (up to 150).

The lower amplitudes of adjusted $\Delta \mathrm{B}_{\mathrm{r}}$ observations in Figure 2 in comparison to Lunar Prospector data in Figure $1 \mathrm{~b}$ (which has $1 \mathrm{M}+$ data points) are primarily related to the higher altitude of two thirds of the dataset. The selection in Figure 2 has $575 \mathrm{~K}+$ along-track differences. The altitude distribution of this SELENE/Kaguya selection is multi-modal, with a natural break in the altitude around $33 \mathrm{~km}$; however, limiting data to altitude of $33 \mathrm{~km}$ led to only $235 \mathrm{~K}+$ data values and thus would not be suitable for mapping global fields.

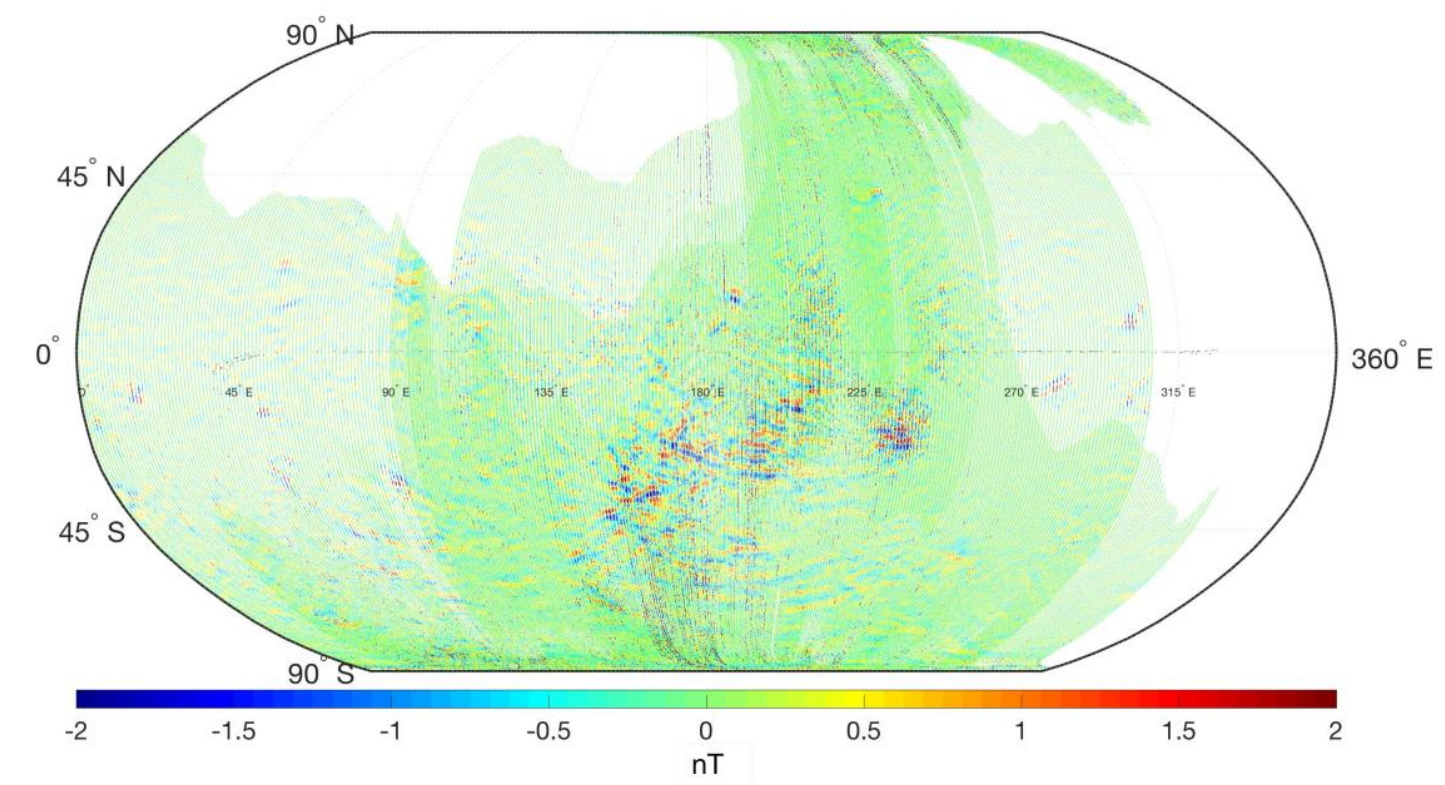

Figure 2. Scatterplot of SELENE/Kaguya extended mission orbit corrected low-altitude ( $\leq$ $45 \mathrm{~km}$ ) along-track differences, $\Delta \mathrm{B}_{\mathrm{r}}$ adjusted in amplitude by 1.25 to account for the 4 second spacing of these observations for amplitude comparison with 5 second LP data in Figure 1b. The selection criteria used are identical to those used for the LP $\Delta \mathrm{B}_{\mathrm{r}}$ shown in Figure 1b. The lower amplitudes of these adjusted $\Delta \mathrm{B}_{\mathrm{r}}$ observations in comparison to Lunar Prospector data in Figure $1 \mathrm{~b}$ (which has $1 \mathrm{M}+$ data points) are primarily related to the related higher altitude of two thirds of the dataset. See text for altitude characteristics of the data.

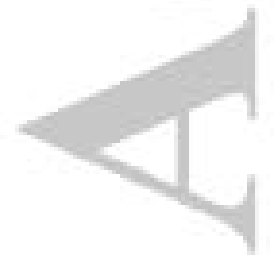




\section{Inversion results}

\subsection{LP and SELENE/Kaguya global inversions}

Using modeling methods described in section 2, we performed equivalent source inversions using vector field observations and their along-track gradients processed with analytical and ad-hoc techniques briefly described in section 3.1. In comparison to models where only along-track gradients were used, models that used vector fields required greater regularization to suppress $\mathrm{N}-\mathrm{S}$ trending along-track artifacts in vector fields, which led to much smoother and smaller amplitude surface field models (see Table 1 and Figure S5 in the supporting information). The artifacts are a result of differing magnitudes of vector fields in neighboring orbits caused by external fields, imperfect corrections, or instrument offsets and are sometimes referred to as biases or local base-level variations.

Each inversion and computation of the model fields took from a few days to 2 weeks of real time on the University of Kentucky High Performance Computing facility and NASA's Pleiades cluster depending on the number of observations used in the inversion. Several tens of trials were performed over a three-year period with different combinations of LP and SELENE/Kaguya and vector and vector gradient datasets and different data preprocessing schemes, data selection criteria, and regularization parameters that used inversions from $35000\left(1^{\circ}\right.$ equal area spacing) monopoles to subset-based global inversions with up to 500,000 monopoles. The most meaningful results from these trials are included in Table 1.

Table 1. Parameters and statistics of different visually stable global models. LP is Lunar Prospector satellite and SVM (Surface Vector Mapping) is Tsunakawa et al. (2010, 2015) method for calculating $B_{\mathrm{r}}$ component of the field at the surface. The statistics of the preferred global model of this study is in boldface.

\begin{tabular}{|c|c|c|c|c|}
\hline Dataset & $\begin{array}{l}\text { Equal-area } \\
\text { monopole spacing } \\
(\text { in degrees } \\
\text { a) }\end{array}$ & $\begin{array}{l}\text { Number of } \\
\text { observations and } \\
\text { mean altitude and } \\
\text { altitude std. dev. } \\
\text { in } \mathrm{km}\end{array}$ & $\begin{array}{l}\text { Damping } \\
\text { parameter } \\
\left(\alpha^{2}\right) \text { of the } \\
\text { selected } \\
\text { model }\end{array}$ & $\begin{array}{l}\text { Global field range } \\
\text { of the final model } \\
\text { at the surface of } \\
\text { the Moon in nT }\end{array}$ \\
\hline $\begin{array}{l}\text { LP only } \\
\text { gradient }\end{array}$ & $1^{\circ}, \sim 30 \mathrm{~km}$ & $\begin{array}{l}1008860 \\
28.8, \quad 7.2\end{array}$ & 7 & $\begin{array}{l}\mathrm{B}_{\mathrm{r}}:\langle-195,+109> \\
\mathrm{B}_{\theta}:\langle-119,+85> \\
\mathrm{B}_{\phi}:\langle-100,+81>\end{array}$ \\
\hline $\begin{array}{l}\text { LP only } \\
\text { Vector and } \\
\text { gradient }\end{array}$ & $1^{\circ}, \sim 30 \mathrm{~km}$ & $\begin{array}{l}\text { Vect: } 1669965 \\
30.0, \quad 7.5 \\
\text { Grad: } 1008860 \\
28.8, \quad 7.2\end{array}$ & $50^{\mathrm{b}}$ & $\begin{array}{l}\mathrm{B}_{\mathrm{r}}:<-195,+152> \\
\mathrm{B}_{\theta}:\langle-132,+99> \\
\mathrm{B}_{\phi}:<-97,+85>\end{array}$ \\
\hline $\begin{array}{l}\text { SELENE } \\
\text { only } \\
\text { gradient }\end{array}$ & $1^{\circ}, \sim 30 \mathrm{~km}$ & $\begin{array}{l}1123379 \\
42.5,11.5\end{array}$ & 10 & $\begin{array}{l}\mathrm{B}_{\mathrm{r}}:\langle-110,+130> \\
\mathrm{B}_{\theta}:\langle-96,+68> \\
\mathrm{B}_{\phi}:\langle-90,+54>\end{array}$ \\
\hline $\begin{array}{l}\text { Selected LP } \\
\text { \& SELENE } \\
\text { Vector }\end{array}$ & $1^{\circ}, \sim 30 \mathrm{~km}$ & $\begin{array}{l}\text { LP: } 724735 \\
28.05,7.35 \\
\text { SELENE: } \\
756239 \\
41.57,12.67\end{array}$ & $50^{b}$ & $\begin{array}{l}\mathrm{B}_{\mathrm{r}}:\langle-193,+150> \\
\mathrm{B}_{\theta}:<-132,+97> \\
\mathrm{B}_{\phi}:<-96,+84>\end{array}$ \\
\hline
\end{tabular}




\begin{tabular}{|c|c|c|c|c|}
\hline $\begin{array}{l}\text { LP only } \\
\text { gradient } \\
(84 \text { subsets })\end{array}$ & $\mathbf{0 . 6 6}^{\circ}, \sim 20 \mathrm{~km}$ & $\begin{array}{l}1008860 \\
28.8, \quad 7.2\end{array}$ & 0.1 & $\begin{array}{l}B_{r}:<-445,+278> \\
B_{\theta}:\langle-190,+284> \\
B_{\phi}:\langle-226,+202>\end{array}$ \\
\hline $\begin{array}{l}\text { LP only } \\
\text { Vector and } \\
\text { gradient } \\
\text { (84 subsets) }\end{array}$ & $0.66^{\circ}, \sim 20 \mathrm{~km}$ & $\begin{array}{l}\text { Vect: } 1669965 \\
30.0,7.5 \\
\text { Grad: } 1008860 \\
28.8, \quad 7.2 \\
\end{array}$ & 0.3 & $\begin{array}{l}\mathrm{B}_{\mathrm{r}}:<-195,+152> \\
\mathrm{B}_{\theta}:<-132,+99> \\
\mathrm{B}_{\phi}:<-97,+85>\end{array}$ \\
\hline $\begin{array}{l}\text { SVM } \\
(230 \\
\text { subsets, } \\
\text { Tsunakawa } \\
\text { et al., 2015) }\end{array}$ & $\begin{array}{l}0.2^{\circ} \text { spacing of } \\
\text { generalized spiral } \\
\text { points (not } \\
\text { equivalent source } \\
\text { spacing) }\end{array}$ & $\begin{array}{l}\text { Vect: } 2002276 \\
\text { Grad: N/A } \\
\text { LP altitude: } \\
\text { 29.41, } 7.90 \\
\text { SELENE altitude: } \\
\text { 42.87, } 12.61\end{array}$ & Variable & $\begin{array}{l}\mathrm{B}_{\mathrm{r}}:\langle-714,+608> \\
\mathrm{B}_{\theta}:\langle-323,+391> \\
\mathrm{B}_{\phi}:\langle-341,+269>\end{array}$ \\
\hline
\end{tabular}

${ }^{a} 1^{\circ}$ latitude on the surface of the Moon is approximately $30 \mathrm{~km}$.

${ }^{\mathrm{b}}$ Very few N-S biases remain in a few regions in this model. High damping parameters in

L1-norm model regularization are needed to overcome N-S biases in some of the passes as shown in Figure S5 in the supporting information.

\subsection{Additional inversion considerations}

From the performance of the Earth's magnetic field models of Olsen et al. (2017), it became clear that the use of along-track gradients alone, i.e., without the use of the vector measurements themselves, could lead to improvement in the modeling of crustal anomaly fields (see also the model simulation that shows the recovery of the field at the surface of the Moon in Figures S1 and S2 in the supporting information). Even without having observations of E-W gradients, such as those available in the Swarm satellite constellation around the Earth, simply from N-S gradients we could obtain models consistent with vector field observations. These models are unaffected by the along-track artifacts known to characterize vector data-based magnetic field models due to external field contamination, without having to apply a strong regularization. Moreover, it should be noted that the along-track observation differences are not purely N-S differences, but also contain small E-W and elevation differences (both typically between 50 and $150 \mathrm{~m}$ ) in the observation locations. However, the contribution of these small differences is certainly not comparable to the advantage of simultaneous gradient observations in all three directions.

The stability of the iteratively reweighted and regularized inversion (section 2.2) depends on the amount and quality of data (in addition to the spacing and depth of equivalent sources). The selection of stable models of each data type listed in Table 1 is based on visual appearance of any deleterious along-track trends or other features indicating noise (examples of this are shown in the supporting information in Figures S4 and S5). Each stable model must also have features consistent with observations. These criteria are necessarily subjective because we do not have any surface fields measured on anomaly features corresponding to features observed at satellite altitudes. Thus, instead of showing unstable and stable models of each data type, we use the range of stably downward continued fields as one of the criteria to decide which model is superior. The logic of this is that if a visually stable model has a larger range of values in the downward continued fields, then that combination of data type and spacing and depth of sources retains more of the signal. The ranges of the stable model fields derived from different permutations and combinations of data are given in Table 1. A larger range in this case implies a higher degree of complexity of the modeled field and the 
range criterion suggest that the use of along-track gradients alone, with subsets, leads to most desirable models. Because these globally derived models made by using single constant damping parameters (that are optimized for regions with least $\mathrm{S} / \mathrm{N}$ ) and larger source spacing are of lower spatial resolution compared to the local models optimized for the $\mathrm{S} / \mathrm{N}$ of specific regions, we compare local models from different approaches where they are derived in section 5.

\subsection{SELENE/Kaguya global vector gradient inversion}

Following the procedures described in section 2, we determined a global model from SELENE/Kaguya along-track gradients. The elevation range of the SELENE/Kaguya extended mission data used is 8-63 km, and low elevations are largely in the longitude range between $90^{\circ}$ and $260^{\circ}$ and in the southern hemisphere. SELENE/Kaguya data-based models required heavier damping parameters for obtaining stable-appearing models which then resulted in a smaller amplitude of the derived components (Table 1).

\subsection{Lunar Prospector subsets-based inversion result}

The stability of global inversions is significantly affected by the number of model parameters and the signal-to-noise ratio $(\mathrm{S} / \mathrm{N})$ which worsens in regions of very small amplitude anomalies. The computer resources available to us (including NASA's Pleiades cluster) did not permit handling many more than 35000 parameters (corresponding to $1^{\circ}$ or $\sim 30 \mathrm{~km}$ spacing) in a global inversion (similar to the LCS-1 model of the Earth's field, Olsen et al., 2017). However, we could significantly improve the spatial resolution of anomaly features and their amplitudes by performing regional inversions. These were relying on subsets of monopoles placed every $0.66^{\circ}$ ( $\sim 20 \mathrm{~km}$ spacing). We chose the subsets so that there is a $10^{\circ}$ overlap with each other. This enabled us to merge the resulting regional models into one global model at the Moon's surface, while avoiding edge effects. The best models derived from radial and total field components with this approach are shown in Figure 3. One cannot use the same damping parameter for all regions of the Moon to create high resolution maps of the Moon because regions with lower $\mathrm{S} / \mathrm{N}$ require larger amount of damping to control noise. If the same high damping parameter is used for the regions with higher $\mathrm{S} / \mathrm{N}$, the highest possible resolution for those regions cannot be achieved.

\subsection{Comparisons with results of Tsunakawa et al. (2015)}

Radial component anomaly features in our maps are also similar to features in the maps of Tsunakawa et al. (2015) which were obtained with inversions of 230 subsets of $0.2^{\circ}$ $(\sim 6 \mathrm{~km})$ spaced basis functions perpendicular to the Moon's surface from Lunar Prospector and SELENE/Kaguya vector components and their along-track differences. In order to maximize the spatial resolution they derived different optimum regularization parameters in different subsets and then recalculated surface $B_{r}$ fields for each subset on a smooth global surface of regularization parameters and finally merged the subsets. They used the Surface Vector Mapping method (Tsunakawa et al., 2014) which allowed them to compute the $B_{r}$ component field at the Moon's surface; they used the $\mathrm{B}_{\mathrm{r}}$ component to compute $\mathrm{B}_{\theta}$ and $\mathrm{B}_{\phi}$ component fields. In their maps, there are a few spurious anomaly features that are neither seen in the observations nor do they appear in our maps (see supporting information Figures S4 and S6). In section 5, comparisons are made of the high resolution regional fields derived 
from this study using monopoles to the ones derived from the SVM approach of Tsunakawa et al. (2015).
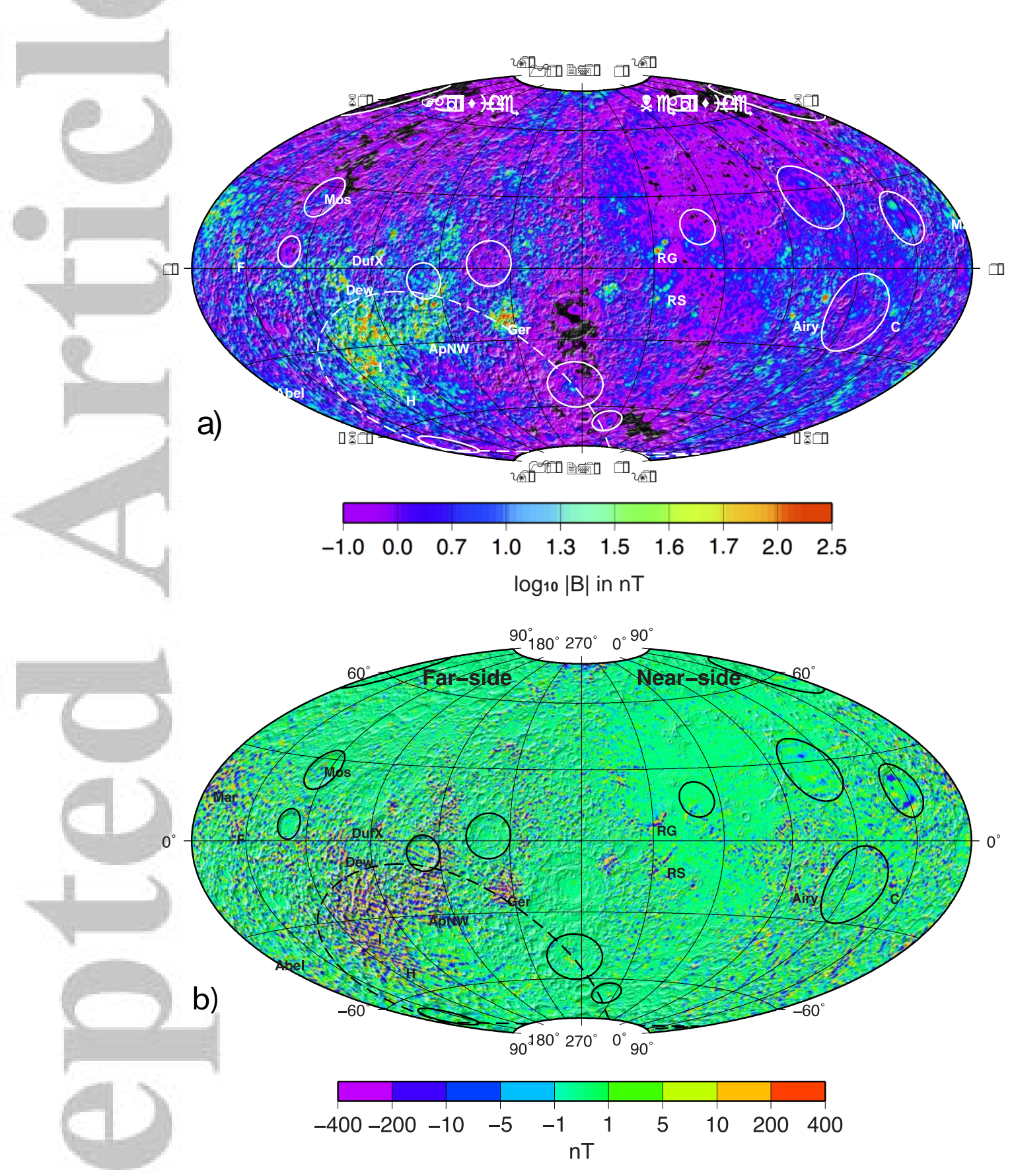

Figure 3. Highest resolution surface magnetic fields from the global monopoles $\left(0.66^{\circ}\right.$ spacing) based models derived in this study from 84 subsets. a) logarithm of total field magnetic anomalies (black color on the map represents regions with total field < $0.1 \mathrm{nT}$ ); b) $\mathrm{B}_{\mathrm{r}}$ component of the field. Data sources as described in section 3.1. Ovals: Nectarian basins; Dashed oval: South Pole - Aitken basin; Magnetic anomalies near swirl features (from left to right): Mar- Marginis, Abel, F- Firsov, Mos - Moscoviense, Dew - Dewar, DufX - Dufay X, I - Ingenii, H - Hopmann, ApNW - NW of Apollo, Ger - Gerasimovich, RG - Reiner Gamma, RS - Rima Sirsalis, Airy, C - Crozier. Hammer-Aitoff projection. 


\subsection{Estimates of variances of spherical harmonic coefficients from global monopoles models}

The comparisons of amplitudes of stably downward continued monopoles based models in Table 1 do not reflect a benefit of including vector component data in the inversions. To understand better how well certain spherical harmonic coefficients are determined from different models, we construct model covariance matrices as discussed by Olsen et al. (2017). The diagonal of the covariance matrices contains variances, $\sigma_{m}^{2}$, of model parameters that can help in the assessment of contributions of different datasets. The covariance matrices, $\mathrm{C}_{\mathrm{m}}$, of spherical harmonic coefficients can only be computed where the G matrix, the full kernel relating model parameters to data locations, is determined from global models. Thus, $\mathrm{C}_{\mathrm{m}}$ could only be determined for our global inversions with 35000 monopoles converted into spherical harmonic coefficients. The determination of covariance matrix of model parameters requires inversion of a matrix that is of the size [model parameters X model parameters] (equation 9 in Olsen et al., 2017) and thus cannot be determined for high degree and orders on any of the computing clusters available to us. Consequently, we determine the covariance matrix for degrees and orders up to 150 (i.e., wavelengths of $>70 \mathrm{~km}$ ). It is important to note that the results of the comparison up to degree/order 150 do not explain the performance of these different datasets at wavelengths shorter than $70 \mathrm{~km}$. Hence, the criterion of rejecting models with $\mathrm{N}-\mathrm{S}$ trending artifacts still outweighs the results of these comparisons.

In Figure 4, we show four low altitude datasets cases: LP selected vector and vector gradient, LP and SELENE/Kaguya selected vector, SELENE/Kaguya vector gradients, and LP vector gradients. The figures show uniform low variances for LP selected vector and vector gradient data and slightly higher variances for low degree and order terms for vector only model. SELENE gradients only model (Figure 4c) has higher variances throughout, whereas LP gradients only model (Figure 4d) has relatively well-determined low degree and order coefficients. In terms of variances of model parameters LP vector and vector gradient data based model has the best performance and thus the use of low altitude LP vector data would be desirable if it were possible to reject orbital segments with large neighboring pass to pass differences in vector component data or develop processing techniques that would eliminate them.

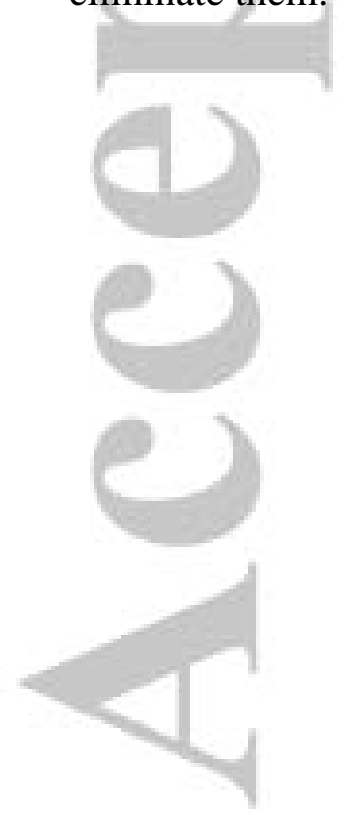



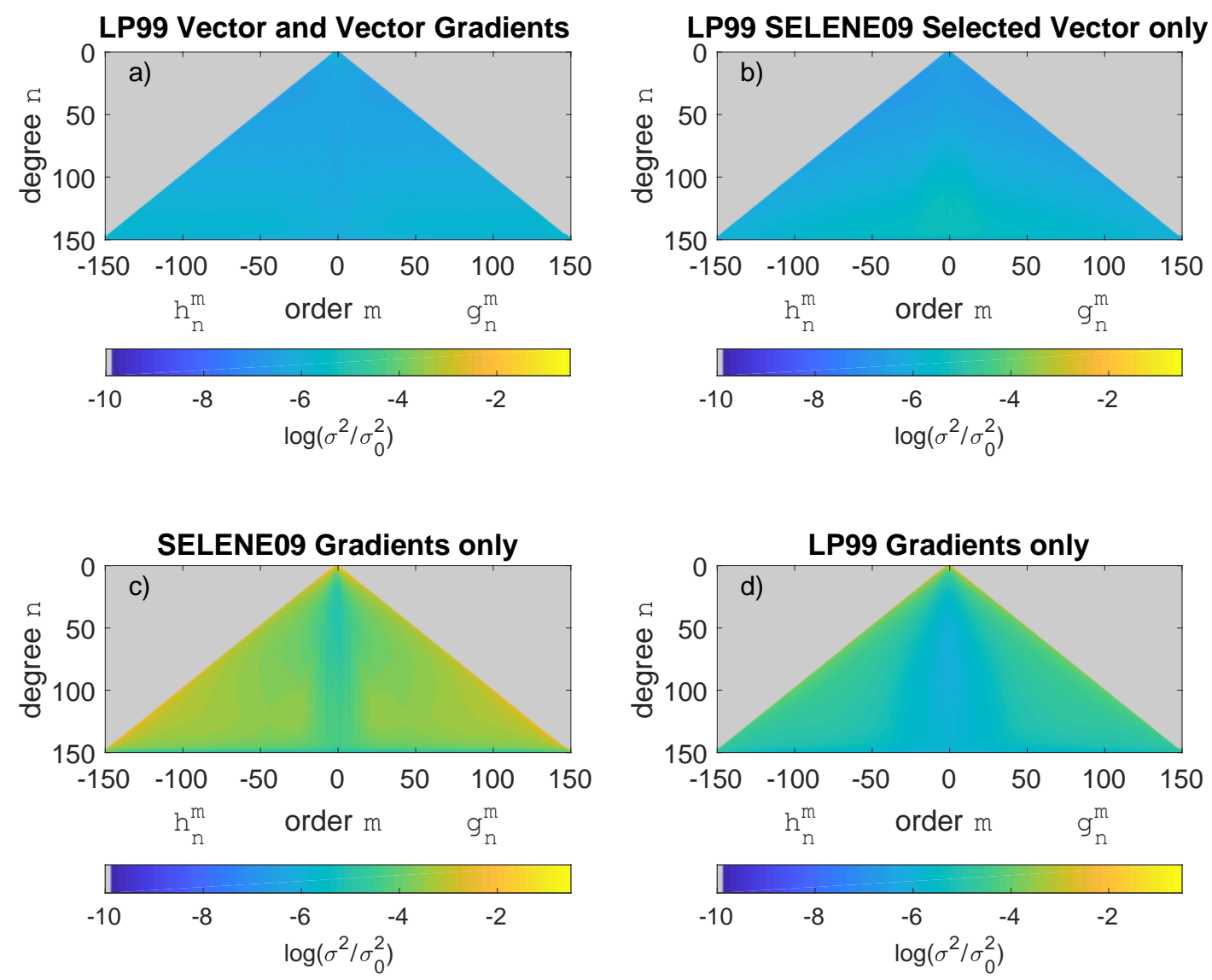

Figure 4. Normalized variances (squared uncertainty) of spherical harmonic coefficients from models using different datasets. a) LP99 (Lunar Prospector low altitude) vector and vector gradient data have the least model variances; b) low order terms in models using LP99 and SELENE09 (SELENE/Kaguya extended mission low altitude) data have higher variances than (a); SELENE09 data based models have higher variances in higher degree and order terms; and LP99 gradients only models have lower variances than (c). We assume uncorrelated data variances $\left(\sigma_{0}{ }^{2}\right.$ $=1$ ); however, the normalization only examines which coefficients are relatively better resolved in models with different datasets. See text for details regarding these comparisons not overriding the criterion of stability of the models at short wavelengths.

Visually, the model based only on LP vector gradient data has primarily the same anomaly features as LP vector and vector gradient model and it also has a greater anomaly amplitude range (see Figure 3 in the manuscript, Figure S5 in the supporting information, and the rightmost column in Table 1), but models based on combined vector and vector gradient data always require higher damping parameters in order to remove obvious anomalous N-S trending features in some regions which also causes smoothing of anomaly features and reduction in their amplitudes (Table 1). Thus, unless problematic orbital pass segments where vector data based models introduce anomalous $\mathrm{N}-\mathrm{S}$ trending features are identified and eliminated (or if their levels can be adjusted), the use of vector data at this juncture should be avoided to obtain maximum spatial resolution of anomaly features at least in equivalent source based downward continued maps. 


\section{Maximizing resolution in local regions}

Improving spatial resolution with monopoles as bases requires smaller source spacing and depth (than $20 \mathrm{~km}$ used for subset global inversions) and it is not possible to achieve this in areas of low $\mathrm{S} / \mathrm{N}$ without higher amount of regularization (which then smooths the features and defeats the purpose). Thus, we chose to maximize spatial resolution only in a few regions of interest and show examples of Reiner Gamma swirl (high anomaly amplitudes), Von Kármán crater (the landing site of Chang'E4 lander in the SPA basin, Huang et al., 2018), and Serenitatis magnetic anomaly (the intended landing site of proposed lunar missions).

\subsection{Reiner Gamma region}

The maximum resolution we could achieve using the LP gradients was in the region of Reiner Gamma swirl (5 km depth monopoles and $7 \mathrm{~km}$ spacing) and it is close to the resolution achieved by Tsunakawa et al. (2015) in the region (see comparisons in Figure 5). Tsunakawa et al. (2015) model also has spurious features marked with red ovals in Figure 5 which are not present in the observations (see supporting information Figure S6 which shows vector components selected in that study).

In Figure 5, we compare two strategies of using monopoles bases with L1-norm model regularization. The fields in the right column are derived in a similar manner to our global modeling strategy: iteratively reweighted L2 model norm minimization, using average of $\mathrm{B}_{\mathrm{r}}^{2}$, followed by L1-norm model regularization using average of $\left|\mathrm{B}_{\mathrm{r}}\right|$ field at the surface. The fields in the center column (our preferred model) are derived using L1-norm model regularization on the residual of fields derived by iteratively reweighted L2-norm minimization. The latter procedure is more tedious but it creates an appearance of fields we are more used to observing in the potential fields modeling (as they have smoother appearance and has fewer isolated features in $\mathrm{Br}$ component that we are uncertain about), while obtaining the sparsity and resolution benefit of L1-norm model regularization. The benefit of our preferred approach can also be surmised by the minimum/maximum range of the derived components at the Moon's surface (comparable to the model of Tsunakawa et al., 2015) shown in Table 2. Based on several studies, the Reiner Gamma region may have been magnetized by an inducing field which was within a few degrees of horizontal and northward direction (e.g., Hood \& Schubert, 1980; Oliveira \& Wieczorek, 2017; Garrick-Bethell \& Kelley, 2019) and thus $\mathrm{B}_{\theta}$ component has the most critical information on its magnetization. In both our models in Figure 5, the $\mathrm{B}_{\theta}$ component appears to display more complex field with similar but more balanced positive-negative range characteristics than Tsunakawa et al. (2015) model. We note that the line and oblong disk source models based on the locations of the dark lanes of the swirl (Hemingway \& Garrick-Bethell, 2012; figure 1a in Garrick-Bethell $\&$ Kelley, 2019) proposed for the Reiner Gamma main magnetic anomaly (centered at $7.5^{\circ} \mathrm{N}$, $302^{\circ} \mathrm{E}$ ) are offset by about $1^{\circ}$ (the center of the disk model is at $7.4^{\circ} \mathrm{N}, 300.9^{\circ} \mathrm{E}$ ) and thus their explanation may need additional unaccounted factors like an eastward dip of the sources or emplacement of magnetic sources away from the sources directly associated with the swirl.

Table 2. Minimum/maximum range and one standard deviation of the surface vector fields from the three different approaches shown in Figure 5. See text for abbreviations of the model names. 


\begin{tabular}{|c|c|c|c|}
\hline $\begin{array}{c}\text { Model/ } \\
\text { Field } \\
\text { Component }\end{array}$ & $\begin{array}{c}\text { Tsunakawa et al. (2015) } \\
\text { SVM model (nT) }\end{array}$ & L2resL1 model (nT) $^{\mathbf{a}}$ & L1 model (nT) $^{\mathbf{b}}$ \\
\hline $\mathrm{B}_{\mathrm{r}}$ & $<-467,+367>$ & $<-426 / 358>$ & $<-810,+651>$ \\
\hline $\mathrm{B}_{\theta}$ & 23.3 & 22.0 & 33.5 \\
\hline $\mathrm{B}_{\phi}$ & $<-148,+334>$ & $<-395,+423>$ & $<-382,+577>$ \\
& 16.5 & 17.3 & $<-383,+489>$ \\
\hline$|\mathbf{B}|$ & 9.6 & $102,+165>$ & 23.6 \\
\hline & $<0,508>$ & 31.5 & $<0,815>$ \\
\hline
\end{tabular}

${ }^{\text {a }}$ L1-norm model regularization on the residual of L2-norm minimization on monopoles bases

${ }^{\text {b }}$ L1-norm model regularization on monopoles bases

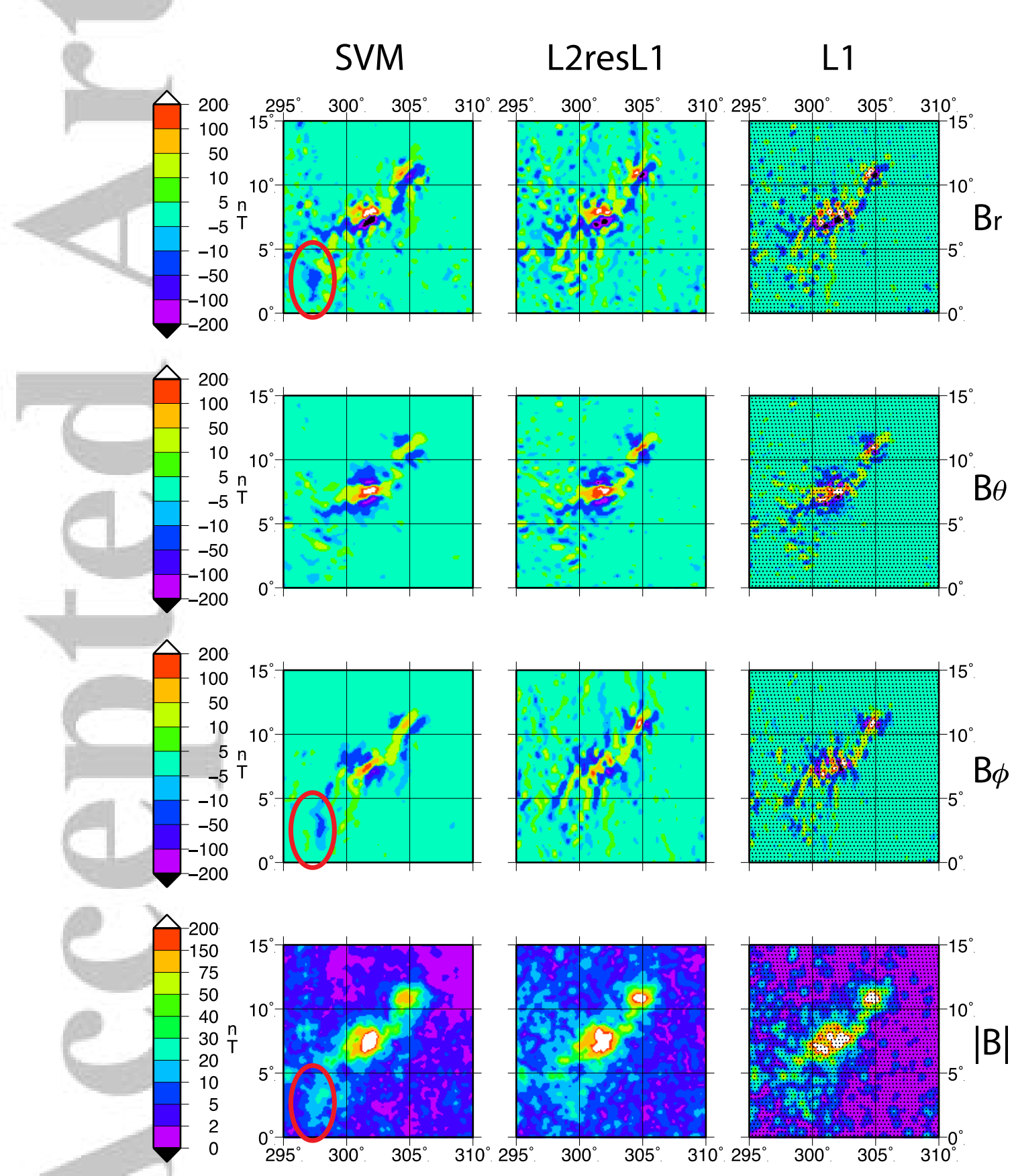


Figure 5. Comparisons of Tsunakawa et al.'s SVM model (left column), and Monopoles L1-norm model regularization on the residual of L2-norm model (L2resL1 model) from LP low altitude along-track differences (central column) and the L1-model norm model (right panel) at the Moon's surface. Black dots on the monopole models show the locations of monopoles $(8.7 \mathrm{~km}$ spacing and 10 $\mathrm{km}$ depth). Tsunakawa et al. model (left) has some spurious features in the southwest corner $\left(297.5^{\circ} \mathrm{E}, 2.5^{\circ} \mathrm{N}\right)$ which are not in the data. The plots of observations and along-track differences are shown in Figure S6 in the supporting information.

\subsection{Von Kármán region}

Figure 6 shows the comparison of Tsunakawa et al. (2015) SVM model, our LP low altitude high resolution model using L1 model norm on the residual of L2 norm (L2resL1), and our SELENE/Kaguya low altitude fields with orbit corrected positions (Goossens et al., 2020 ) processed using L1 model norm based field model. East of longitude $175^{\circ} \mathrm{E}$, the amplitudes are low in Tsunakawa et al. field (left panels) and the SELENE/Kaguya low altitude fields (right panels); the LP low altitude data are less subdued (central panels) and so the lower amplitudes in models that use SELENE/Kaguya datat must arise from those data. Figure S7 in supporting information shows that the eastern part of the SVM and L1 (SELENE*) models in Figure 6 are based on primarily a few high altitude passes data and thus has much lower amplitudes, whereas usable LP along-track difference data in this study has low altitude passes and has relatively higher amplitudes in the eastern part of the model.

The SELENE/Kaguya data based model (right panels) has NW-SE trending doublet in the $\mathrm{B}_{\mathrm{r}}$ component in the southern part (labeled A in Figure 6) that is not observed in the corresponding LP based data (central panels) and a number of SE-NW trends in the $\mathrm{B}_{\theta}$ component in the vicinity of the above identified features. It is difficult to ascertain, without examining all of the low altitude data and examining other geological and geophysical information, whether the alignment of these features is from improvements due to orbit corrections of the SELENE/Kaguya extended mission data (Goossens et al., 2020).

Other than the issues of the high altitude SELENE data in the eastern part of the region and the doublet like features mentioned above, the three models have many similar features. L1 (SELENE*) model is sparse and where possible it has intensifies amplitudes associated with certain monopole regions at the expense of regions surrounding them. In all total field maps in Figure 6, Von Kármán crater appears to have an area of low magnetic field to the SE (near the star of Chang'e 4 lander site). The center of Leibnitz crater has a magnetic high in the total field maps (labeled L in the central panel) which is surrounded by a low magnetic region. The low to the east of the crater is intensified in the two maps that use SELENE data (i.e., primarily high altitude data). Each of these models is derived using different methodologies and datasets and the differences are reasonable especially in the downward continued fields shown in Figure 6.

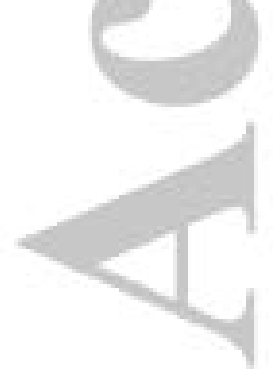



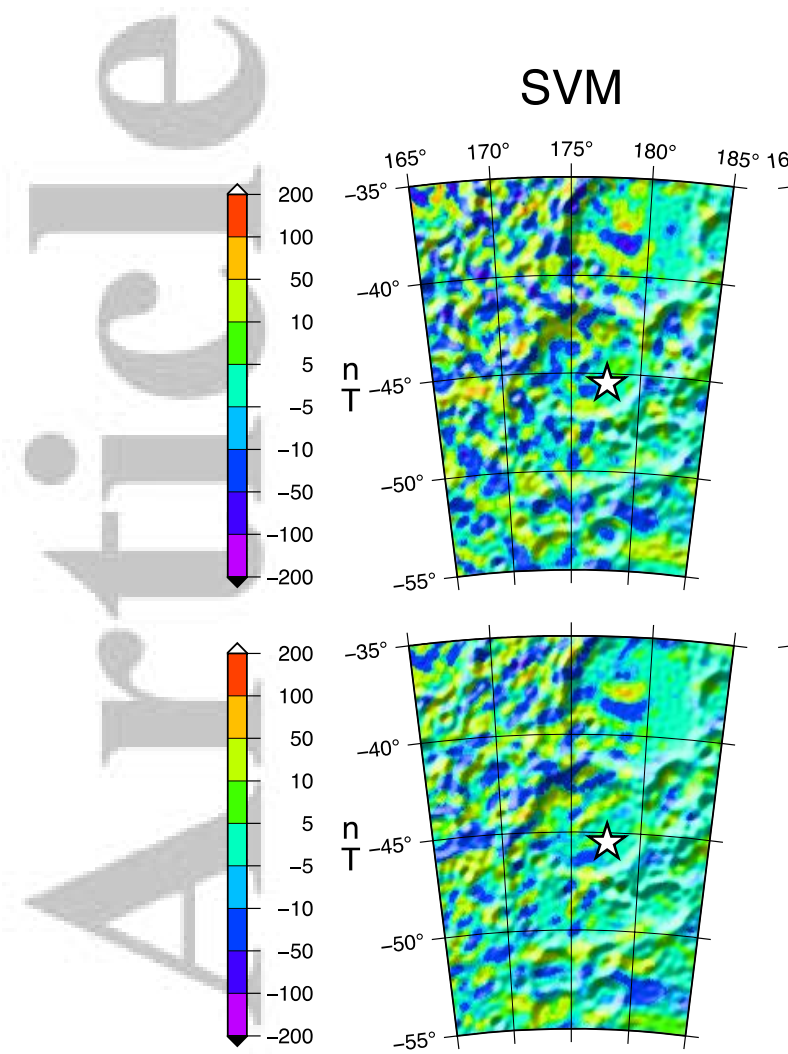

L2ResL1 (LP) L1 (SELENE*)
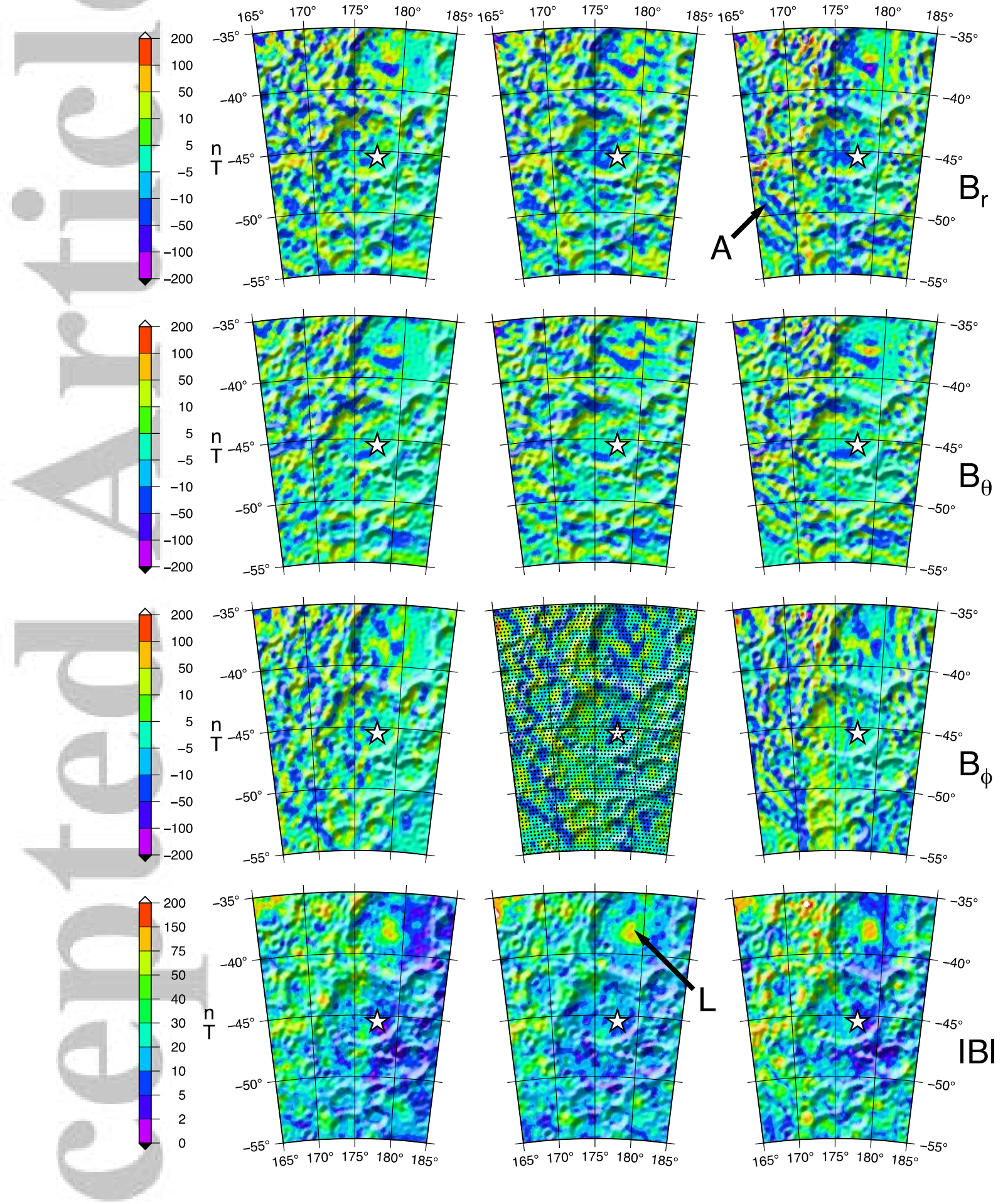

Figure 6. Von Kármán crater region comparison between the fields at the surface of the Moon from three different models. The SVM model of Tsunakawa et al. (2015) (left panels), monopoles based L2resL1 model of this study from LP low altitude along-track differences (central panels), and L1 (SELENE*) is the L1 monopoles model with SELENE data with low altitude extended orbit corrected positions (Goossens et al., 2020) (right panels). Labels A and $\mathrm{L}$ are described in the text. The orbit corrections have not substantially changed SELENE/Kaguya field patterns. White stars show the location of Chang'E4 lander. Black 
dots in the L2resL1 column $\mathrm{B}_{\phi}$ component are the locations of equivalent sources; these are shown to illustrate that small features on these maps are smoothly varying and formed by multiple sources and thus it is unlikely that they could be artifacts.

\section{Results over the Serenitatis (northern) anomaly from monopoles vs dipoles approach}

A comparison of high- resolution downward continued fields derived from the equivalent source monopoles and dipoles approaches would be of significant interest. We thus implemented the equivalent-dipoles-based L1 model norm regularization on the gradients of vector fields. We noted in section 2.1 that the fields from dipoles bases function can only be derived stably at larger source spacing due to deleterious interactions between neighboring dipoles (Langlais et al., 2004; Mayhew, 1979; Ravat et al., 1991). Here, we compare the two approaches for a relatively strong magnetic feature in the Serenitatis basin. Figure 7 shows a comparison of surface fields for the stable monopoles bases at $20 \mathrm{~km}$ depth and at $7 \mathrm{~km}$ spacing and (center panels), the most stable fields we could derive from the dipoles approach (20 km depth at $20 \mathrm{~km}$ spacing (right panels), and the SVM model fields (left panels). The along-track differences associated with the principal Serenitatis magnetic feature (not shown) are relatively clean (the heavy black rectangles in the center of the maps), but the region also has a number of short-wavelength variations which the inversion with the dipoles bases in unable to capture.

One can also use the characteristics of the bipolar nature of components, the spread of the components, and their sign to infer the magnetization direction in simple cases. The equivalent-dipole-based fields (i.e., a single negative $\mathrm{B}_{\mathrm{r}}$ component lobe and bipolar positive (to the North)/negative (to the South) pattern of $\mathrm{B}_{\theta}$ component is characteristic of a nearly vertically downwardly magnetized source (see p. 77-78 in Blakely, 1995, for the patterns of the components from a dipole in local magnetic coordinate system, i.e., $Z=-B_{r}$ and $X=-B_{\theta}$ ). If the $\mathrm{B}_{\mathrm{r}}$ field were to originate from a dipole or a sphere, its maximum depth-to-the-center determined from the width of the feature $\left(2^{\circ}\right.$ or $\left.\sim 60 \mathrm{~km}\right)$ would be approximately $20 \mathrm{~km}$ using the relationship between the width of the $\mathrm{B}_{\mathrm{r}}$ feature and depth to the dipole given in Blakely (1995),

depth $=\frac{\text { width }}{2 \sqrt{2}}$.

The $\mathrm{B}_{\theta}$ component feature also has positive/negative lobes consistent with nearly vertically downwardly magnetized source (see figure 4.9c in Blakely, 1995). The relationship between the distance of the positive/negative peaks of the $\mathrm{B}_{\theta}$ component, i.e., the depth of an equivalent dipole $=$ the distance between the positive and negative peaks (Blakely, 1995), however, leads to the depth of about $60 \mathrm{~km}$. The disparity between the two depth estimates and the observation that the positive/negative lobes of $\mathrm{B}_{\theta}$ component are separated unlike in the case of a dipole shown in Blakely (1995) and are also extended in the E-W direction makes it clear that the source region is not a simple compact magnetized body. Forward modeling such a feature without any constraints on the source geometry is non-unique (e.g., a number of sources near the surface could approximate the field equally well as different configurations of deeper sources). Thus, here we only outline a few reasonable inferences from the modeled vector components.

In addition to the broader Serenitatis anomaly features discussed above, there are also a few shorter wavelength dipole-like anomaly features in the central and right panels, and 
because they have bipolar patterns in either $\mathrm{B}_{\mathrm{r}}$ or $\mathrm{B}_{\theta}$ field maps, they too appear to be due to magnetic sources and not noise in the data as short-wavelength variations over these features are present in the along-track differences. For example, the positive/negative pair in the $\mathrm{B}_{\theta}$ field in the western part of the main Serenitatis source region, which has a corresponding negative feature in the $\mathrm{B}_{\mathrm{r}}$ field, could be a near vertically downwardly magnetized source similar to the main Serenitatis source region. Similarly, the northward extension of the main Serenitatis positive/negative bipolar feature in the $\mathrm{B}_{\theta}$ field may have been caused by a vertically downward magnetized source near $33^{\circ} \mathrm{N}, 18.5^{\circ} \mathrm{E}$, an expression of which seen in the monopoles-based $\mathrm{B}_{\mathrm{r}}$ field (top-central panel). Depth estimates are disparate for even these smaller sources (with the above two formulas for compact sources) and suggest that even this short-wavelength feature is caused by more complex sources than a compact dipole-like source.

The main features of the components in the SVM model are somewhat different and in some cases have different orientations in comparison to the main features on our dipolesbased model from LP along-track differences (Figure 7). In a separate study (L. Cole \& D. Ravat, manuscript under preparation), the best-fitting magnetic moment orientations were derived using the method of Oliveira \& Wieczorek (2017), which determines magnetic moment directions of dipoles situated on a plane (a condition less stringent than the seamount problem of Parker, 1991, which requires the knowledge of the source geometry or at least the upper surface of the source geometry). The use of LP along-track differences directly instead of the SVM vector fields model (to reduce the effect of noise in the vector data) leads to a more near-vertical magnetization direction (dipole moment inclination of $70^{\circ}-80^{\circ}$, L. Cole \& D. Ravat, manuscript under preparation). High positive inclinations imply that the negative pole of the planetocentric dipole (conventionally called north paleopole) that magnetized the region must be closer to the location of the anomaly (than the paleopole of Oliveira \& Wieczorek, 2017), and thus will fall well in the region of nearside impacts. Taking all of these above characteristics and results into account, the principal Serenitatis anomaly feature is very likely caused by a coalescence of anomalies from many smaller sources but with magnetization consistent with near-vertically downward directed magnetized sources.

Not all of the relatively short-wavelength anomalies in the region appear to be magnetized in a near-vertically downward direction. For example, the positive $\mathrm{B}_{\mathrm{r}}$ feature at $34^{\circ} \mathrm{N}, 21^{\circ} \mathrm{E}$ has a corresponding bipolar feature (negative to the North and positive to the South) and that implies upwardly directed magnetization. On the other hand, the positive/negative pair in the $\mathrm{B}_{\mathrm{r}}$ field near $34^{\circ} \mathrm{N}, 17.5^{\circ} \mathrm{E}$ has a corresponding positive feature in the $\mathrm{B}_{\theta}$ component field and two negative side-lobes to its north and south and thus the magnetic source of this feature could be interpreted to have a near horizontal and northward directed magnetization (see figure 4.9b in Blakely, 1995). It is possible that such differently magnetized smaller sources could have been caused by a combination of later impact demagnetization and shock related changes in the magnetization direction (Gattacceca et al., 2010; Tikoo et al., 2015) or may have formed by thermal remanent magnetization when the region was elsewhere with respect to the orientation of the core field than the primary Serenitatis sources analyzed here. While oppositely directed magnetization can be achieved in a reversal, sources in a region having both vertical and horizontal magnetization implies a large true polar wander if these directions are not altered through another mechanism (like a later impact shock during the dynamo epoch). In general, if a feature has no bipolar (antisymmetric/asymmetric) pattern in either $\mathrm{B}_{\mathrm{r}}$ or $\mathrm{B}_{\theta}$ component however, then the feature could be considered noise. 
To sum up our examination of the Serenitatis region magnetic variations, there are many short-wavelength bipolar features mapped in the surface magnetic fields in this study that may not be due to noise in the data. For many anomaly features, the spatial resolution of the downward continued maps is of wavelengths in the range of $25-30 \mathrm{~km}$ based on the width of positive or negative features (approximately half-the-wavelength). The main Serenitatis anomaly features are likely a coalescence of anomalies of several near vertically downward magnetized sources which implies the region was near the north magnetic pole if the Moon's dynamo was dipolar (Arkani-Hamed \& Boutin, 2017; Weiss \& Tikoo, 2014). Because the feature is at $30^{\circ}-35^{\circ} \mathrm{N}$ latitude presently, in itself this would imply a significant true polar wander on the Moon since the formation of the Serenitatis magnetic sources. Finally, the closer spacing of monopoles in the inversion has afforded a higher resolution mapping of the field than the SVM model which uses vector data with noise or the dipole-based modeling of the along-track differences of the vector components.

Oliveira \& Wieczorek (2017) determined magnetization directions of several magnetic anomaly features on the Moon which yielded paleopoles that appear to avoid Procellarum KREEP Terrane (PKT); however, if our above inference of the near-vertical magnetization direction of the principal sources of Serenitatis feature is correct, then at least the paleopole of this source may lie within the PKT. Oliviera \&Wieczorek (2017) analyzed the SVM model fields at $30 \mathrm{~km}$ altitude and the resulting SVM vector components have different trends and locations of maxima and minima than the monopoles or dipoles based fields (see Figure 7). Hence, the magnetization directions determined from that model will be different. Moreover, there are a couple of other smaller magnetic features we have examined in the region that appear to be magnetized in near-vertical reversed and approximately horizontal directions. If the inference of near-vertically and near-horizontally magnetized sources in the region is correct, then the Serenitatis region itself indicates significant true polar wander. However, based on the examination of one region, we cannot judge the validity of Oliveira \&Wieczorek's (2017) observation that their paleopoles could be within a few tens of degrees of $90^{\circ} \mathrm{W}$ and $90^{\circ} \mathrm{E}$ longitudes and avoid the PKT.

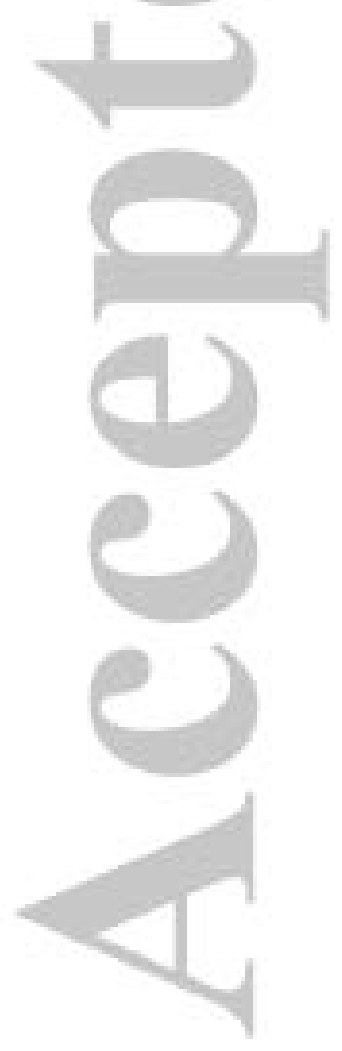




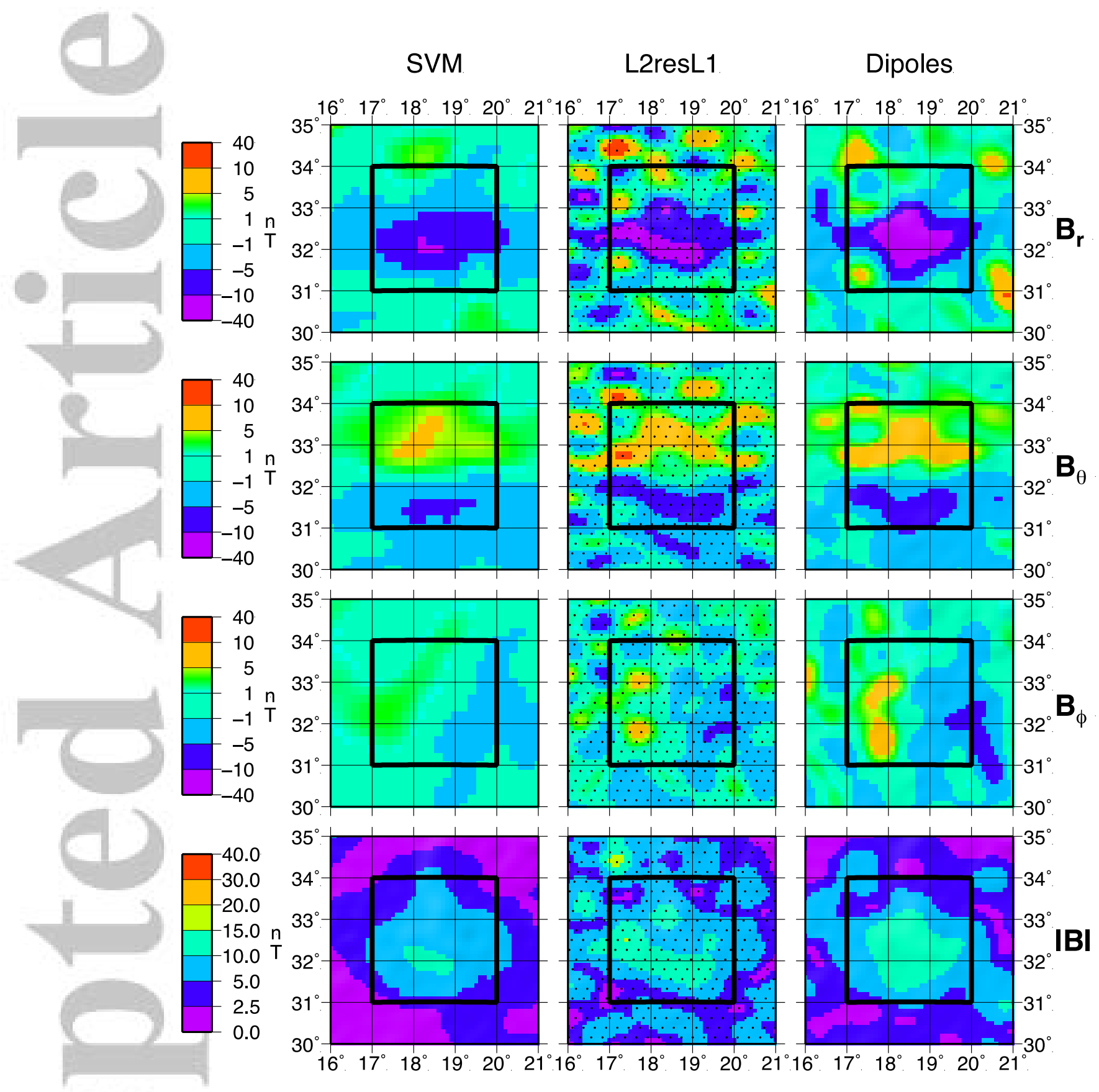

Figure 7. Serenitatis magnetic anomaly comparisons at the surface of the Moon between (left) the SVM model (Tsunakawa et al., 2015) and our L1 norm monopoles model on the residual of L2 norm (L2resL1 model) (middle) and dipoles (right) basis functions models optimized for the primary anomaly feature (shown by black box). The SVM range for $\mathrm{B}_{\mathrm{r}}$ is $\langle-10.4,+8.2\rangle \mathrm{nT}$, the L2resL1model shows a number of detailed features and the $B_{r}$ range of the primary feature is $<-34$, $+35.3>\mathrm{nT}$ and the comparable stable dipoles model's $\mathrm{B}_{\mathrm{r}}$ range is $<-14,+5.2>\mathrm{nT}$. The locations of $7 \mathrm{~km}$ spacing monopoles are shown in the center panel using black dots. The SVM fields $\mathrm{B}_{\theta}$ and $\mathrm{B}_{\phi}$ components have different trends than the monopoles or dipoles fields and the magnetization directions determined from that model will be different. 


\section{Other data selection and processing considerations for the future}

There are a number of mission characteristic issues that require further attention in order to improve the utility of these datasets, but are beyond the scope of this study. These are: While the magnetometers on Lunar Prospector and SELENE/Kaguya were triaxial fluxgate magnetometers, there were significant differences in the mission that probably impact how the data should be combined. In the case of Lunar Prospector, the spacecraft was spin stabilized at $\sim 12 \mathrm{rpm}$, and the spin axis was approximately perpendicular to the celestial equator for the low altitude part of the mission. Because we average the magnetic field over a spin period, any magnetic field biases associated with the two axes perpendicular to the spin axis will be corrected. The axes parallel to the spin axis has to be calibrated in other ways, and in Lunar Prospector these included calibrations by comparison to the predicted magnetic field in the terrestrial magnetosphere, and calibrations using Alfven waves outside of the terrestrial magnetosphere.

The SELENE/Kaguya was a three-axis stabilized spacecraft with magnetometer at the end of a long (12 m) mast, which was initially stowed in a canister and deployed after orbit insertion (Tsunakawa et al., 2010). The momentum wheels failed during the lowest altitude part of the mission, and so the thrusters were engaged for attitude control. This suggests that the mast/boom may have experienced some significant movement or swaying relative to the spacecraft body, where the attitude is determined. The attitude errors would have to be large, in the range of several degrees or more, to significantly affect the accuracy of the vector components. We do not know if including only the SELENE/Kaguya scalar field, the most well-determined part of the lunar magnetic field, would help in reconciling the SELENE/Kaguya and Lunar Prospector magnetic field measurements. The accuracy of the location information from the lowest altitude part of SELENE's mission was of the order of kilometers, while the accuracy of the LP's location is of the order of meters (Goossens et al., 2020).

\section{Conclusions}

We used several permutations and combinations of data, data reduction strategies, and magnetic field modeling approaches (discussed in sections 2 and 3) to derive models of magnetic field vector components at the lunar surface. We found that magnetic fields can be globally downward continued well by using along-track vector gradients of data collected in the wake region of the Moon with respect to the impinging solar wind. Downward continuation was accomplished with regularization using L1-norm model regularization minimizing the average of $\left|\mathrm{B}_{\mathrm{r}}\right|$ component at the lunar surface. We found that inclusion of vector component data in modeling led to $\mathrm{N}-\mathrm{S}$ trends in the derived fields where the vector fields differed in neighboring orbits. These N-S trending artifacts could only be suppressed using relatively large regularization (which led to unacceptable amount of smoothing and amplitude reduction of genuine crustal anomaly signal). On the other hand, when only alongtrack gradient data were used, it was possible to derive cleaner downward continued field maps with smaller amount of regularization. Our highest resolution global fields computed from regional subset-based inversions, using equivalent source monopoles (O'Brien \& Parker, 1994; Olsen et al., 2017) at $20 \mathrm{~km}$ spacing and at $20 \mathrm{~km}$ depth, have similar features as the maps of Tsunakawa et al. (2015). The monopole solutions are more stable at closer spacing and depths than allowed by dipoles as basis functions. 
It was possible to improve the resolution as well as amplitudes of anomaly features where the crustal field signal is relative large and noise from external fields is low. Such modeling over Reiner Gamma swirl (a region of high S/N) suggests that the monopoles approach can significantly improve the resolution and amplitudes of magnetic features when monopoles are placed shallow $(\sim 5 \mathrm{~km})$ and closer $(\sim 7 \mathrm{~km})$. The L1-norm model regularization however generates sparse models where amplitudes are concentrated in fewer sources and thus the resulting fields have spotty appearance (high amplitudes surrounded by large near zero fields). To make smooth appearing non-sparse, yet high resolution field models, we applied L1-norm model regularization on the residual of an L2-norm regularized model. This procedure also led to surface fields of higher amplitudes and spatial resolution of better than $30 \mathrm{~km}$ wavelength than our models discussed earlier. At this point, without having more regional near-surface magnetic measurements, we cannot judge whether the local high resolution models should be sparse or not (or how sparse they should be). No downward continuation procedure with observational and round-off errors will recover short-wavelength signal that has reached below the noise threshold at observation altitude.

Despite improvements in the positions of orbits of the SELENE/Kaguya satellite extended mission (Goossens et al., 2020), there is no clear evidence of improvement in the mapped anomaly features. In inversions with the orbit corrected data, continuity of features is improved in and around the region of Von Kármán impact crater (in comparison to the SVM model) and a few interesting anomaly doublets have formed (that are not observed in the Lunar Prospector based models), further close examination is needed in additional regions before the orbit corrected SELENE/Kaguya low altitude extended mission data can be combined with the LP data to generate field models.

One important interpretive result is related to the magnetization direction derived from the field of the magnetic anomaly feature situated in the Serenitatis crater with equivalent source dipoles. The $\mathrm{B}_{\mathrm{r}}$ and $\mathrm{B}_{\theta}$ anomaly patterns of this feature form a classic textbook pattern arising from a near-vertically downward pointing magnetized source. This interpretation is different than the more inclined magnetization derived by Oliveira \& Wieczorek (2017). Nonetheless, the real situation in the region is quite complex as evident from the patterns of fields from monopoles inversions with L1 model norm. The Serenitatis L1 iterations on the residual of L2 (L2resL1) monopoles solution suggests that the sources in the region are more complexly distributed than suggested by the SVM or our stable dipole model. The vector component fields from our dipole and L2resL1 models have better defined anomaly patterns that are consistent with each other than the patterns of the SVM model.

\section{Acknowledgments and Data}

We appreciate the great efforts of Lunar Prospector and SELENE/Kaguya teams for collecting valuable data analyzed in this study. We thank Ian Garrick-Bethell, an anonymous reviewer, and editors for their meticulous reviews. We are grateful to Sander Goossens and Erwan Mazarico of Goddard Space Flight Center for making available orbit positioning improved magnetic data from the SELENE/Kaguya extended mission. An undergraduate research student working with DR, Lillie Cole, generously allowed us to include her key result of modeling the Serenitatis magnetic feature prior to publication. We thank Kimberly Moore for discussions related to elastic net based sparse models. We also thank comments and suggestions on the manuscript made by Aspen Davis, Brooks Rosandich, and Ratheesh Kumar R. T. DR is grateful for the support from the NASA research grant NNX16AN51G 
which made this work possible. All of our preferred global and local models of magnetic field at the lunar surface are available in Ravat et al. (2020) provided in references.

\section{References}

Arkani-Hamed, J. \& Boutin, D. (2014). Analysis of isolated magnetic anomalies and magnetic signatures of impact craters: Evidence for a core dynamo in the early history of the Moon, Icarus, doi: http://dx.doi.org/10.1016/j.icarus.2014.04.046

Arkani-Hamed, J. \& Boutin, D. (2017). South Pole Aitken Basin magnetic anomalies: Evidence for the true polar wander of Moon and a lunar dynamo reversal, J. Geophys. Res. Planets, 122, 1195-1216, doi:10.1002/2016JE005234

Backus, G., Parker, R. \& Constable, C. (1996). Foundations of Geomagnetism: Cambridge University Press.

Blakely, R. (1995). Potential theory in gravity and magnetic applications: Cambridge University Press.

Collinson, D. W., Stephenson, A., \& Runcorn, S. K. (1973). Magnetic properties of Apollo 15 and 16 rocks. Geochimica Et Cosmochimica Acta, 3, 2963-2976.

Daily, W. D., \& Dyal, P. (1979). Theories for the origin of lunar magnetism. Phys. Earth Planet. Int., 20, 255-270.

Denevi, B. W., Robinson, M. S., Boyd, A. K., Blewett, D. T., \& Klima, R. L. (2016). The distribution and extent of lunar swirls. Icarus, 273, 53-67. doi:10.1016/i.icarus.2016.01.017

de Pater, I., \& Lissauer, J. J. (2015). Planetary Sciences (Updated Second Edition ed.): Cambridge University Press.

Dyal, P., Parkin, C. W., \& Daily, W. D. (1974). Magnetism and the Interior of the Moon. Reviews of Geophysics and Space Physics, 12(4), 568-591.

Dyment, J., \& Arkani-Hamed, J. (1998). Equivalent Source Magnetic Dipoles Revisited. Geophys. Res. Lett., 25(11), 2003-2006.

Garrick-Bethell, I., and Kelley, M. R. (2019). Reiner Gamma: A Magnetized Elliptical Disk on the Moon, Geophys. Res. Lett., 46, 5065-5074, doi: 10.1029/2019GL082427

Gattacceca, J., Boustie, M., Lima, E., Weiss, B. P., de Resseguier, T., \& Cuq-Lelandais, J. P. (2010). Unraveling the simultaneous shock magnetization and demagnetization of rocks. Phys. Earth Planet. Int., 182, 42-49.

Goossens, S., Mazarico, E., Ishihara, Y., Archinal, B.A., Gaddis, L. (2020). Improving the geometry of Kaguya extended mission data through refined orbit determination using laser altimetry, Icarus, 336, doi:10.1016/j.icarus.2019.113454.

Haines, G. V. (1985). Spherical cap analysis. J. Geophys. Res., 90, 2583-2591.

Hemingway, D., \& Garrick-Bethell, I. (2012). Magnetic field direction and lunar swirl morphology: Insights from Airy and Reiner Gamma. J. Geophys. Res., 117, E10012. doi:10.1029/2012JE004165

Hood, L. L., \& Schubert, G. (1980). Lunar Magnetic Anomalies and Surface Optical Properties. Science, 208, 49-51.

Hood, L. L., Zakharian, A., Halekas, J., Mitchell, D. L., Lin, R. P., Acuña, M. H., \& Binder, A. B. (2001). Initial mapping and interpretation of lunar crustal magnetic anomalies using Lunar Prospector magnetometer data. J. Geophys. Res., 106(E11), 27825-27839.

Kiefer, W. S., Macke, R. J., Britt, D. T., Irving, A. J., \& Consolmagno, G. J. (2012). The density and porosity of lunar rocks. Geophys. Res. Lett., 39. doi:10.1029/2012GL051319 
Kother, L., Hammer, M. D., Finlay, C. C., \& Olsen, N. (2015). An equivalent source method for modelling the global lithospheric magnetic field. Geophys. J. Int., 203(1), 553-566.

Langel, R. A., \& Hinze, W. J. (1998). The magnetic field of the Earth's lithosphere : the satellite perspective. Cambridge ; New York: Cambridge University Press.

Langlais, B., Purucker, M., \& Mandea, M. (2004). Crustal magnetic field of Mars. $J$. Geophys. Res., 109(E02008), doi:10.1029/2003JE002048.

Leopardi, P., 2006. A partition of the unit sphere into regions of equal area and small diameter, Electronic Transactions on Numerical Analysis, 25(12), 309-327.

Lepaulard, C., Gattacceca, J., Uehara, M., Rochette, P., Quesnel, Y., Macke, R. J., \& Kiefer, S. J. W. (2019). A survey of the natural remanent magnetization and magnetic susceptibility of Apollo whole rocks. Phys. Earth Planet. Int., 290, 36-43. doi:10.1016/j.pepi.2019.03.004

Lin, R. P., Mitchell, D. L., Curtis, D. W., Anderson, K. A., Carlson, C. W., McFadden, J., . . . Binder, A. (1998). Lunar Surface Magnetic Fields and Their Interaction with the Solar Wind: Results from Lunar Prospector. Science, 281, 1480-1484. doi:10.1126/science.281.5382.1480

Maus, S. (2010). An ellipsoidal harmonic representation of Earth's lithospheric magnetic field to degree and order 720. Geochem. Geophys. Geosyst., 11, Q06015. doi:10.1029/2010GC003026

Maus, S., Rother, M., Holme, R., L“uhr, H., Olsen, N., \& Haak, V. (2002). First scalar magnetic anomaly map from CHAMP satellite data indicates weak lithospheric field. Geophys. Res. Lett., 29(14), 47. doi:10.1029/2001GL013685

Mayhew, M. A. (1979). Inversion of satellite magnetic anomaly data. J. Geophys., 45, 119128.

Nagata, T., Fisher, R. M., Schwerer, F. C., Fuller, M. D., \& Dunn, J. R. (1971). Magnetic properties and remanent magnetization of Apollo 12 lunar materials and Apollo 11 lunar microbreccia. Proceedings of the Second Lunar Science Conference, 3, 24612476.

O’Brien, M. S., \& Parker, R. L. (1994). Regularized geomagnetic field modelling using monopoles. Geophy. J. Int., 118, 566-578.

Oliveira, J. S., \& Wieczorek, M. A. (2017). Testing the axial dipole hypothesis for the Moon by modeling the direction of crustal magnetization. J. Geophys. Res. Planets, 122. doi:10.1002/2016JE005199

Oliveira, J. S., Wieczorek, M.A., \& Kletetschka, G. (2017) Iron abundances in lunar impact basin melt sheets from orbital magnetic field data, J. Geophys. Res. Planets, 122, 2429 2444. doi: 10.1002/2017JE005397

Olsen, N., Ravat, D., Finlay, C. C., \& Kother, L. K. (2017). LCS-1: a high-resolution global model of the lithospheric magnetic field derived from CHAMP and Swarm satellite observations. Geophy. J. Int., 211, 1461-1477. doi:10.1093/gji/ggx381

Parker, R. L. (1991), A Theory of Ideal Bodies for Seamount Magnetism, J. Geophys. Res., 96, 16101-16112.

Purucker, M., \& Nicholas, J. B. (2010). Global spherical harmonic models of the internal magnetic field of the Moon based on sequential and coestimation approaches. $J$. Geophys. Res., 115, E12007. doi:10.1029/2010JE003650.

Purucker, M., Head, J. W. \& Wilson, L. (2012). Magnetic signature of the lunar South PoleAitken basin: Character, origin, and age. J. Geophys. Res., 117, E05001. doi:10.1029/2011JE003922.

Ravat, D. (2011). Interpretation of Mars southern highlands high amplitude magnetic field with total gradient and fractal source modeling: New insights into the magnetic mystery of Mars. Icarus, 214, 400-412. doi:10.1016/j.icarus.2011.05.004. 
Ravat, D.; Purucker, M. E.; \& Olsen, N. (2020) "New Magnetic Field Models of the Moon". Earth and Environmental Sciences Research Data. 2. doi:https://doi.org/10.13023/9wpett68,https://uknowledge.uky.edu/ees_data/2

Ravat, D., Hinze, W. J., \& von Frese, R. R. B. (1991). Lithospheric magnetic property contrasts within the South American plate derived from damped least-squares inversion of satellite magnetic data. Tectonophysics, 192, 159-168.

Runcorn, S. K., Collinson, D. W., O" Reilly, W., Stephenson, A., Greenwood, N. N., \& Battey, M. H. (1970). Magnetic Properties of Lunar Samples. Science, 167, 697-699.

Sharp, L. R., Coleman, P. J., Lichtenstein, B. R., Russell, C. T., \& Schubert, G. (1973). Orbital mapping of the lunar magnetic field. The Moon (7), 322-341. doi:10.1007/BF00564638

Strangway, D. W., Larson, E. E., \& Pearce, G. W. (1970). Magnetic Properties of Lunar Samples. Science, 167, 691-693.

Takahahshi, F., Shimizu, H., Matsushima, M., Shibuya, H., Matsuoka, A., Nakazawa, S., .. . Tsunakawa, H. (2009). In-orbit calibration of the lunar magnetometer onboard SELENE (KAGUYA). Earth, Planets and Space, 61, 1269-1274.

Thébault, E. (2008). A proposal for regional modelling at the Earth's surface, R-SCHA2D. Geophy. J. Int., 174(1), 118-134.

Thébault, E., Mandea, M., \& Schott, J. J. (2006). Modeling the lithospheric magnetic field over France by means of revised spherical cap harmonic analysis (R-SCHA). $J$. Geophys. Res., 111, B05102. doi:10.1029/2005JB004110

Tikoo, S. M., Gattacceca, J., Swanson-Hysell, N. L., Weiss, B. P., Suavet, C., \& Cournède, C. (2015). Preservation and detectability of shock-induced magnetization, J. Geophys. Res. Planets, 120, 1461-1475, doi:10.1002/2015JE004840

Tsunakawa, H., Takahahshi, F., Shimizu, H., Shibuya, H., \& Matsushima, M. (2015). Surface vector mapping of magnetic anomalies over the Moon using Kaguya and Lunar Prospector observations. J. Geophys. Res. Planets, 120, 1160-1185. doi:10.1002/2014JE004785

Tsunakawa, H., Shibuya, H., Takahahshi, E., Shimizu, H., Matsushima, M., Matsuoka, A., . . . Iijima, Y. (2010). Lunar Magnetic Field Observation and Initial Global Mapping of Lunar Magnetic Anomalies by MAP-LMAG Onboard SELENE (Kaguya). Space Science Reviews, 154, 219-251. doi:10.1007/S31214-010-9652-0

von Frese, R. R. B., Hinze, W. J., \& Braile, L. W. (1981a). Spherical Earth gravity and magnetic anomaly analysis by equivalent point source inversion. Earth Planet. Sci. Lett., 53, 69-83.

von Frese, R. R. B., Hinze, W. J., Braile, L. W., \& Luca, A. J. (1981b). Spherical earth gravity and magnetic anomaly modeling by Gauss_Legendre quadrature integration. $J$. Geophys. Res., 49, 234-242.

Weiss, B. P., \& Tikoo, S. M. (2014). The lunar dynamo. Science, 346. doi: $10.1126 /$ science. 1246753

Wieczorek, M. A. (2018). Strength, depth, and geometry of magnetic sources in the crust of the Moon from localized power spectrum analysis. Journal of Geophysical Research: Planets, 123, 291-316. doi:https://doi.org/10.1002/2017JE005418

Wieczorek, M. A., Weiss, B. P., \& Stewart, S. T. (2012). An Impactor Origin for Lunar Magnetic Anomalies. Science, 335. doi:10.1126/science.1214773

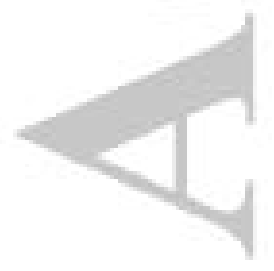

